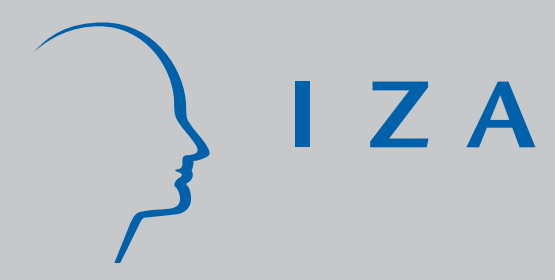

IZA DP No. 955

Risky Human Capital Investment, Income Distribution, and Macroeconomic Dynamics

Volker Grossmann

December 2003 


\title{
Risky Human Capital Investment, Income Distribution, and Macroeconomic Dynamics
}

\author{
Volker Grossmann \\ University of Zurich \\ and IZA Bonn
}

\section{Discussion Paper No. 955 \\ December 2003}

\author{
IZA \\ P.O. Box 7240 \\ D-53072 Bonn \\ Germany \\ Tel.: +49-228-3894-0 \\ Fax: +49-228-3894-210 \\ Email: iza@iza.org
}

This Discussion Paper is issued within the framework of IZA's research area The Future of Labor. Any opinions expressed here are those of the author(s) and not those of the institute. Research disseminated by IZA may include views on policy, but the institute itself takes no institutional policy positions.

The Institute for the Study of Labor (IZA) in Bonn is a local and virtual international research center and a place of communication between science, politics and business. IZA is an independent, nonprofit limited liability company (Gesellschaft mit beschränkter Haftung) supported by Deutsche Post World Net. The center is associated with the University of Bonn and offers a stimulating research environment through its research networks, research support, and visitors and doctoral programs. IZA engages in (i) original and internationally competitive research in all fields of labor economics, (ii) development of policy concepts, and (iii) dissemination of research results and concepts to the interested public. The current research program deals with (1) mobility and flexibility of labor, (2) internationalization of labor markets, (3) welfare state and labor market, (4) labor markets in transition countries, (5) the future of labor, (6) evaluation of labor market policies and projects and (7) general labor economics.

IZA Discussion Papers often represent preliminary work and are circulated to encourage discussion. Citation of such a paper should account for its provisional character. A revised version may be available on the IZA website (www.iza.org) or directly from the author. 
IZA Discussion Paper No. 955

December 2003

\section{ABSTRACT}

\section{Risky Human Capital Investment, Income Distribution, and Macroeconomic Dynamics*}

This paper analyzes the interaction between intergenerational wealth transmission, human capital investments under uninsurable labor income risk, and economic growth in a small open overlapping-generations economy with heterogeneous agents. It demonstrates how the role of the personal income distribution for an economy's process of development through risky human capital accumulation depends on the shape of the saving function. Consistent with recent empirical evidence, the analysis suggests that the impact of higher inequality on the aggregate human capital stock, and thus, on growth may be positive. This result rests on two features of the model, which both are largely supported by empirical evidence. First, as shown under weak conditions, children's human capital investments are positively affected by parents' income. Second, the marginal propensity to save is increasing in income.

JEL Classification: $\quad$ I20, O11, O40

Keywords: growth, income distribution, intergenerational transfers, risky education, saving function

Volker Grossmann

Socioeconomic Institute

University of Zurich

Zürichbergstr. 14

8032 Zürich

Switzerland

Tel.: +4116342 288

Email: volker.grossmann@wwi.unizh.ch

\footnotetext{
* I am grateful to Josef Falkinger and Omer Moav for detailed comments and suggestions on a previous version of the paper. Moreover, I am grateful to Johannes Binswanger, Panu Poutvaara, Josef Zweimüller and seminar participants at the University of Zurich for helpful discussions.
} 


\section{Introduction}

This paper analyzes the interaction between intergenerational wealth transmission, human capital investments under uninsurable labor income risk, and economic growth in a small open overlapping-generations economy with heterogeneous agents. It demonstrates how the role of the personal income distribution for an economy's process of development through risky human capital accumulation depends on the shape of the saving function.

The analysis suggests that despite diminishing individual returns to educational investments the impact of higher inequality on the aggregate human capital stock and thus on growth may be positive during the transition to a stationary equilibrium. This result rests on two features of the model, which both are largely supported by empirical evidence. First, children's human capital investments are positively affected by parents' income. ${ }^{1}$ For advanced countries, this evidence is surprising at the first glance because credit constraints for human capital investments do not seem to be binding for most individuals (or are, at least, negligible). ${ }^{2}$ However, uninsurable labor income risk systematically affects incentives of risk-averse individuals to invest in human capital, an effect which has received surprisingly little attention in the growth literature. ${ }^{3}$ For instance, individuals face idiosyncratic and nondiversifiable risk associated with labor demand shocks for specific skills. Moreover, among other sources, they face health and disability risk, uncertainty about the quality of schooling, and risk regarding access to social networks and other social factors which affect individual labor market prospects. The second crucial ingredient of the model which gives rise to a potentially positive relationship between inequality and growth is that the marginal propensity to save is

\footnotetext{
${ }^{1}$ Empirical studies have controlled for parents' education, occupation and ability of both children and parents, still finding a positive and substantial effect of parents' income on human capital investments (e.g., Taubman, 1989; Sacerdote, 2002; Plug and Vijverberg, 2003).

${ }^{2}$ Mainly, because government lending for educational purposes largely overcomes this market failure.

${ }^{3}$ See Gould et al. (2001), Bénabou (2002) and Krebs (2003) for notable exceptions, which, however, are concerned with different questions. Gould et al. (2001) are concerned with the evolution of wage inequality and its interaction with technical progress. Bénabou (2002) examines the trade-offs of progressive income taxation for growth and efficiency, whereas Krebs (2003) studies the impact of labor income risk on growth in a framework with ex ante identical agents.
} 
increasing in income (e.g., Menchik and David, 1983; Dynan et al., 2000, 2002). According to the model, adults save in order to bequeath or to make inter vivos gifts, respectively. ${ }^{4}$ Intergenerational transfers are optimally allocated to human capital investments and savings for future wealth of the young. ${ }^{5}$ With an increasing marginal propensity to save, higher inequality implies that the average reduction in wealth transmission of the rich may outweigh the average increase in wealth transmission of the poor despite diminishing individual returns to educational investments. Consequently, aggregate human capital investment may fall.

The question how the personal income distribution affects an economy's process of development has stimulated both macroeconomic theory and growth empirics in the last decade like almost no other one. Whereas earlier empirical evidence suggests a negative link between inequality and growth (e.g., Alesina and Rodrik, 1994; Persson and Tabillini, 1994; Perotti, 1996), using a new and comprehensive high-quality data set, Deininger and Squire (1998) and Banerjee and Duflo (2003) find practically none, whereas Li and Zou (1998) and Forbes (2000) report a positive relationship. Interestingly, allowing for a non-linear impact of inequality on GDP growth, Barro (2000) finds a negative relationship for developing countries and a positive relationship for more advanced countries.

A positive relationship between inequality and growth in advanced countries is difficult to reconcile with more recent theoretical approaches in the literature on income distribution and macroeconomics. ${ }^{6}$ In their pioneering work, Galor and Zeira (1993) show that inequality typically has an adverse effect on the process of development if credit markets are imperfect ("credit-market imperfections approach"). This is because poor individuals cannot borrow sufficiently high amounts to finance an indivisible level of schooling investments. ${ }^{7}$ Hence, the credit-market imperfections approach is consis-

\footnotetext{
${ }^{4}$ Such a "joy of giving" saving motive has received strong empirical support. See Carroll (2000) for an illuminating discussion of the empirical evidence.

${ }^{5}$ The basic structure of the model builds on Galor and Moav (2004) in a way discussed throughout the paper.

${ }^{6}$ For surveys of this literature, see e.g. Aghion et al. (1999) and Grossmann (2001, ch. 1).

${ }^{7}$ As shown by Bénabou (1996) and Moav (2002), a negative relationship between inequaliy and growth can also be obtained by replacing this non-convexity in the education technology by the
} 
tent with evidence of a negative relationship between inequality and growth in developing countries. In contrast, in the theory proposed in this paper, individuals do not face any borrowing constraints to finance educational investments. Thus, the present model is particularly capable to shed light into the relationship between inequality and growth in advanced countries. Both the credit-market imperfections approach and the theory proposed in the present paper may thus be viewed as complementary. ${ }^{8}$

The mechanism underlying a potentially positive effect of inequality on growth proposed by the theory developed in this paper provides an alternative to the classical view, which argues that wealth inequality is positively related to investment-driven growth. ${ }^{9}$ The foundation of the classical view by Bourguignon (1981) shows that under an increasing marginal propensity to save, unegalitarian stable equilibria are even Pareto superior to an egalitarian stable equilibrium in the neoclassical growth model of Stiglitz (1969). The reason for this result is that physical capital accumulation, fueled by domestic savings, raises wages such that growth "trickles down" to less wealthy individuals. This mechanism is excluded in the present small open economy framework.

assumption of diminishing marginal returns to human capital investment. This implies that the aggregate human capital stock increases if educational investment is spread more equally. Another strand of literature deals with the role of imperfect capital markets for the relationship between wealth distribution and entrepreneurship, when project sizes (i.e., required physical capital investments to become entrepreneur) are fixed (e.g., Banerjee and Newman, 1991, 1993; Aghion and Bolton, 1997).

${ }^{8}$ Besides the credit market imperfections approach, it has been argued that high inequality is adversely related to growth because it induces high demand for redistributive taxation in the political process (e.g., Alesina and Rodrik, 1994; Persson and Tabellini, 1994), high fertility (e.g., Perotti, 1996), high social instability (e.g., Venieris and Gupta, 1986; Alesina and Perotti, 1996), low aggregate demand for R\&D-intensive products (Zweimüller, 2000), and a low degree of specialization of labor (Fishman and Simhon, 2002). Some other contributions, although suggesting a positive relationship between inequality and growth, are relevant for developing contries only. For instance, if capital markets are imperfect and borrowing constraints are binding even for the rich (i.e., in very poor countries), redistribution to the rich enables more individuals to finance education (e.g., Perotti, 1993; Moav, 2002). Moreover, Galor and Tsiddon (1997) argue that for relatively poor economies, equality in the distribution of human capital may be an impedient to prosperity in the longer run under two conditions: first, the individuals' level of human capital positively depends on the parental level of human capital and, second, technological progress depends on the average level of human capital in the economy.

${ }^{9}$ Galor and Moav (2004) offer a unified approach which combines the classical view and the creditmarket imperfections approach. Hypothesizing a co-linear saving function in a closed economy model, they show that inequality and growth are positively related in early stages of development when physical capital accumulation is the prime engine of growth, but are negatively related in mature stages of development when human capital accumulation is the prime engine of growth and borrowing constraints are still binding for the poor. 
Rather, the model offers a mechanism which accounts for the crucial role of human capital accumulation under idiosyncratic human capital risk in modern societies. It shows that even in a small open economy, in which national savings are unrelated to physical capital investment, the relationship between inequality and an economy's the process of development critically depends on intergenerational wealth transmission and thus on savings behavior.

The paper is organized as follows. Section 2 presents the basic structure of the model. Section 3 analyzes individual education and saving decisions. Section 4 examines the role of inequality for aggregate income dynamics. Section 5 discusses the main ingredients and results of this paper in the light of empirical evidence. The last section concludes. Some technicalities as well as an illustrative example are relegated to an appendix.

\section{The Model}

Consider a small open overlapping-generations economy with uninsurable risk of educational investments.

\subsection{Production of Final Output}

In every period, a single homogenous consumption good is produced according to a neoclassical, constant-returns-to-scale production technology. Output at time $t, Y_{t}$, is

$$
Y_{t}=F\left(K_{t}, H_{t}\right) \equiv H_{t} f\left(k_{t}\right), \quad k_{t} \equiv K_{t} / H_{t}
$$

where $K_{t}$ and $H_{t}$ are the amounts of physical capital and human capital employed in period $t$, the latter being measured in efficiency units. $f(\cdot)$ is a strictly monotonic increasing and strictly concave function which fulfills $\lim _{k \rightarrow \infty} f^{\prime}(k)=0$ and $\lim _{k \rightarrow 0^{+}} f^{\prime}(k)=\infty$.

Output is sold to the world market in a perfectly competitive environment, with output price normalized to unity. The rate of return to capital, $r_{t}$, is internationally 
given and time-invariant, i.e., $r_{t}=\bar{r}$. Thus, profit maximization of the representative firm in any period $t$ implies that $k_{t}$ is given by $\bar{r}=f^{\prime}\left(k_{t}\right)$. Thus, $k_{t}=\left(f^{\prime}\right)^{-1}(\bar{r}) \equiv \bar{k}$. Consequently, the wage rate per efficiency unit of human capital, $w_{t}$, reads $w_{t}=$ $f(\bar{k})-\bar{k} f^{\prime}(\bar{k}) \equiv \bar{w}$. Moreover, since $Y_{t}=H_{t} f(\bar{k})$ in this economy, $Y_{t}$ (i.e., the gross domestic product) grows at the same rate as the aggregate human capital stock $H_{t} \cdot{ }^{10}$

\subsection{Individuals and Education Technology}

In each period, there is a unit mass of individuals with two-period lives. In the first period, individuals live by their parents and devote their entire time to acquire education. In the second period (adulthood), individuals supply their efficiency units of human capital to the labor market, and allocate their income between consumption and transfers to their offspring (i.e., bequests or inter vivos transfers, respectively). Intergenerational transfers (i.e., savings of adults) are optimally allocated (either by parent or child) between human capital investment and savings of the young for future wealth. ${ }^{11}$ Individuals are identical with respect to their preferences and their ability to acquire human capital, but may differ in family wealth. So far, this overlappinggenerations structure follows Galor and Moav (2004). However, in contrast to their model, individuals face idiosyncratic human capital risk. Moreover, in order to focus on this aspect and to make the analysis particularly applicable to advanced countries in which credit constraints to finance higher education seem to play a minor role, I assume that individuals can freely borrow for educational purposes, e.g. due to government lending. ${ }^{12}$ (In contrast, developing countries do not have this type of institution, and thus are not focus of the present analysis.) However, as a matter of fact, even in advanced countries human capital risk is uninsurable (e.g. Arrow, 1971), which is

\footnotetext{
${ }^{10}$ The capital-skill complementarity underlying production function (1) is empirically well supported; see e.g. Goldin and Katz (1998). This technology is common in the literature on income distribution, human capital and growth.

${ }^{11}$ Human capital investments can be thought of both schooling and nonschooling forms of training.

${ }^{12}$ The present model also differs to Galor and Moav (2004) in that our small open economy assumption excludes the feedback mechanism from aggregate savings to factor prices which also underlies the results in Bourguignon (1981). Moreover, in order to study the role of savings behavior for the relationship between inequality and growth, no particular functional form on utility is imposed.
} 
therefore assumed.

An individual $i$ born in period $t$ (a member $i$ of generation $t$ ) with investment $e_{t}^{i}$ (in units of the consumption good) in education obtains

$$
h_{t+1}^{i}=h\left(e_{t}^{i}, \tilde{a}\right)
$$

efficiency units of human capital. $\tilde{a}$ is a random variable which follows an i.i.d. process and is drawn each period from a (cumulative) distribution function $\Phi(\tilde{a})$ with support $\mathcal{A}=[\underline{a}, \bar{a}] \subset \mathbb{R}$. The random shock realizes only after investment decisions are made, i.e., in the beginning of the second period of life. The function $h(e, a)$ fulfills the following properties.

A1. For all $a \in \mathcal{A}, h_{e}(e, a)>0, h_{e e}(e, a)<0, h_{a}(e, a)>0, h_{e a}(e, a)>0$, $\lim _{e \rightarrow \infty} h_{e}(e, a)=0$ and $\lim _{e \rightarrow 0^{+}} h_{e}(e, a)=\infty$.

( $h_{e}$ denotes the first partial derivative of $h$ with respect to $e$, etc.) $h_{e e}<0$ implies that expected marginal returns to educational investment are diminishing. ${ }^{13}$ Moreover, given that $h_{a}>0$ which merely serves as a convention, $h_{e a}>0$ implies that the variance of earnings increases with human capital investment $e .{ }^{14}$ Finally, the latter two conditions in A1 ensure interior solutions with respect to educational investment decisions. ${ }^{15}$

Denote by $s_{t}^{i}$ and $b_{t}^{i}$ the amount of savings invested in the financial market and the

\footnotetext{
${ }^{13}$ This reflects the fact that "human capital is inherently embodied in humans and the existence of physiological constraints subjects its accumulation at the individual level to diminishing returns" (Galor and Moav, 2004, p. ??).

${ }^{14}$ See Levhari and Weiss (1974) for a discussion of this assumption and supporting empirical evidence. For more recent evidence, see Pereira and Martins (2002, 2003). A similar type of risk also underlies the model of Bénabou (2002). There are other notions of labor income risk. For instance, Gould et al. (2001) argue that an increasing variance of sectoral shocks increase educational attainment of workers which differ in ability because general education reduces the costs of moving across sectors.

${ }^{15}$ The modelling strategy to assume that human capital investment is riskier than physical capital investment seems plausible (Krebs, 2003). First, human capital risk is nondiversifiable since embodied in individuals, whereas diversified portfolios of financial capital can be held. Second, many forms of financial assets in advanced countries are indeed almost risk-free (e.g. government bonds).
} 
amount of wealth received by member $i$ of generation $t$, respectively, i.e., $s_{t}^{i}=b_{t}^{i}-e_{t}^{i}$. Thus, income of member $i$ of generation $t$ as an adult is given by

$$
I_{t+1}^{i}=\bar{w} h_{t+1}^{i}+\bar{R} s_{t}^{i}=\bar{w} h\left(e_{t}^{i}, \tilde{a}\right)+\bar{R}\left(b_{t}^{i}-e_{t}^{i}\right) \equiv I\left(b_{t}^{i}, e_{t}^{i}, \tilde{a}\right),
$$

where $\bar{R} \equiv 1+\bar{r}$. Utility $U_{t}^{i}$ of member $i$ of generation $t$ is given by a utility function $u$ which is defined over consumption $c_{t+1}^{i}$ as an adult and transfer $b_{t+1}^{i}$ to her offspring ("joy of giving"), i.e.,

$$
U_{t}^{i}=u\left(c_{t+1}^{i}, b_{t+1}^{i}\right)
$$

A2. $u_{c}>0, u_{b}>0, u_{c c}<0, u_{c c} u_{b b}-\left(u_{c b}\right)^{2}>0, u_{c b} \geq 0$ and $\lim _{I \rightarrow \infty} u_{c}(I, 0)<$ $\lim _{I \rightarrow \infty} u_{b}(I, 0)$.

Thus, $u$ is strictly monotonic increasing and strictly concave, which, as will become apparent below, implies risk aversion of individuals. Moreover, the latter two relations in A2 imply normality of intergenerational transfers $b_{t+1}^{i}$, which is the empirically relevant case.

Finally, assume that there are two groups of dynasties in the initial period $t=0$. A fraction $\lambda \in(0,1)$ of ("rich") young individuals in $t=0$ receives a transfer $b_{0}^{R}>0$ and a fraction $1-\lambda$ of ("poor") individuals receives a transfer $b_{0}^{P} \in\left[0, b_{0}^{R}\right)$ from their parent. Thus, in the aggregate, an amount $B_{0} \equiv \lambda b_{0}^{R}+(1-\lambda) b_{0}^{P}$ is initially transferred. Adult individuals possess an aggregate human capital stock $H_{0}$ in the initial period, i.e., initial output is $Y_{0}=H_{0} f(\bar{k}){ }^{16}$

\footnotetext{
${ }^{16}$ Introducing endogenous growth, e.g. by assuming that the aggregate human capital stock $H_{t}$ enters the education technology as positive externality (following Glomm and Ravikumar, 1992, among others), i.e., letting $h_{t+1}^{i}=h\left(e_{t}^{i}, \tilde{a}, H_{t}\right)$, does not alter the main insights of this paper. The main focus lies on the impact of initial inequality (in $t=0$ ) on subsequent growth averaged over a longer period (i.e. on $Y_{t} / Y_{0}-1$, and thus on $Y_{t}, t \geq 1$ ), in order to address the empirical literature on inequality and growth (see section 5.1 for a discussion). Introducing endogenous growth through human capital accumulation simply implies that initial inequality also affects the period-by-period growth rate $Y_{t} / Y_{t-1}-1$ in a qualitatively similar fashion as $Y_{t}$ both during transition to a stationary equilibrium and in the long run.
} 


\section{Individual Decisions}

Note that income $I_{t+1}^{i}=I\left(b_{t}^{i}, e_{t}^{i}, \tilde{a}\right)$ of an adult member $i$ of generation $t$ is a random variable ex ante, but is known in period $t+1$ (i.e., after realization of the shock) when allocating income to consumption and transfer to her offspring. The budget constraint of such an individual in $t+1$ reads $c_{t+1}^{i}+b_{t+1}^{i} \leq I_{t+1}^{i}$. Thus, under the additional constraint $b_{t+1}^{i} \geq 0$, her optimal transfer in $t+1$ is given by

$$
b\left(I_{t+1}^{i}\right) \equiv \underset{b_{t+1}^{i} \geq 0}{\arg \max } u\left(I_{t+1}^{i}-b_{t+1}^{i}, b_{t+1}^{i}\right) .
$$

$b(I)$ is called "saving function" (as $b_{t+1}^{i}$ equals forgone consumption of an adult) and has the following properties.

Lemma 1. Under A2. There exists an income level $\underline{I} \geq 0$ such that $b\left(I_{t+1}^{i}\right)>0$ and $b^{\prime}\left(I_{t+1}^{i}\right)>0$ for all $I_{t+1}^{i}>\underline{I}$.

Proof. Note that, according to (5), $b_{t+1}^{i}$ is implicitly given by the first-order condition

$$
\Omega\left(b_{t+1}^{i}, I_{t+1}^{i}\right) \equiv-u_{c}\left(I_{t+1}^{i}-b_{t+1}^{i}, b_{t+1}^{i}\right)+u_{b}\left(I_{t+1}^{i}-b_{t+1}^{i}, b_{t+1}^{i}\right) \leq 0
$$

which holds with equality if $b_{t+1}^{i}>0$. According to (6), $\Omega_{b}=u_{c c}-2 u_{c b}+u_{b b}$ and $\Omega_{I}=-u_{c c}+u_{c b}$, i.e., A2 implies $\Omega_{I}>0$ and $\Omega_{b}<0 .{ }^{17}$ First, suppose $b_{t+1}^{i}=0$. Since $\Omega_{I}>0$, the left-hand side of the inequality in (6) is strictly increasing in $I_{t+1}^{i}$. Hence, using $\lim _{I \rightarrow \infty} u_{c}(I, 0)<\lim _{I \rightarrow \infty} u_{b}(I, 0)$ from A2, eventually, $b_{t+1}^{i}>0$ if income $I_{t+1}^{i}$ exceeds some level $\underline{I} \geq 0$. Second, for $b_{t+1}^{i}>0$, applying the implicit function theorem reveals

$$
b^{\prime}(I)=-\frac{\Omega_{I}}{\Omega_{b}}=\frac{u_{c c}-u_{c b}}{u_{c c}-2 u_{c b}+u_{b b}}>0
$$

under A2.

From the optimal allocation of income earned as an adult, we can derive the fol-

${ }^{17}$ To confirm $\Omega_{b}<0$, use $u_{c c}<0$ and $u_{c c} u_{b b}-\left(u_{c b}\right)^{2}>0$ from A2 to obtain $u_{b b}<\left(u_{c b}\right)^{2} / u_{c c}$. Thus, $\Omega_{b}=u_{c c}-2 u_{c b}+u_{b b}<u_{c c}-2 u_{c b}+\left(u_{c b}\right)^{2} / u_{c c}=\left(u_{c c}-u_{c b}\right)^{2} / u_{c c}<0$. Of course, this is nothing else than showing that strict concavity of $u$ implies strict quasiconcavity of $u$. 
lowing properties of indirect life-time utility,

$$
v\left(I_{t+1}^{i}\right) \equiv u\left(I_{t+1}^{i}-b\left(I_{t+1}^{i}\right), b\left(I_{t+1}^{i}\right)\right) .
$$

Lemma 2. Under A2. $v(I)$ is a strictly monotonic increasing and strictly concave function.

Proof. First, if $b_{t+1}^{i}=b\left(I_{t+1}^{i}\right)=0$, we have $v\left(I_{t+1}^{i}\right)=u\left(I_{t+1}^{i}, 0\right)$; thus, $v^{\prime}(I)=$ $u_{c}(I, 0)>0$ and $v^{\prime \prime}(I)=u_{c c}(I, 0)<0$. If $b(I)>0$, then $v^{\prime}(I)=u_{c}(I-b(I), b(I))>0$, according to (8), (6) and the envelope theorem. Thus, $v^{\prime \prime}(I)=u_{c c}+b^{\prime}(I)\left(u_{c b}-u_{c c}\right)$. Substituting (7) into the latter expression, we obtain $v^{\prime \prime}(I)=u_{c c}-\left(u_{c b}-u_{c c}\right)^{2} /\left(u_{c c}-\right.$ $\left.2 u_{c b}+u_{b b}\right)$. Manipulating the latter expression implies $v^{\prime \prime}(I)=\left[u_{c c} u_{b b}-\left(u_{c b}\right)^{2}\right] / \Omega_{b}$ (recall $\Omega_{b}=u_{c c}-2 u_{c b}+u_{b b}<0$ ). Hence, $v^{\prime \prime}(I)<0$, according to the strict concavity of $u(c, b)$ presumed (assumption A2). This concludes the proof.

According to Lemma 2, individuals are risk-averse. Throughout the remainder of the paper, the widely accepted assumption of "decreasing absolute risk aversion" is maintained.

A3. $A(I) \equiv-v^{\prime \prime}(I) / v^{\prime}(I)$ is strictly decreasing in $I .^{18}$

An amount of transfers $b_{t}^{i}$ received by a member $i$ of generation $t$ is allocated to savings for future wealth, $s_{t}^{i}$ (with a safe return $\bar{R}$ ), and the risky investment in education, $e_{t}^{i}$, to maximize expected life-time utility $E\left[v\left(I_{t+1}^{i}\right)\right]=E\left[v\left(I\left(b_{t}^{i}, e_{t}^{i}, \tilde{a}\right)\right)\right]$, where $E$ is the expectation operator. Thus, using (3), the optimal human capital investment is given by

$$
e\left(b_{t}^{i}\right) \equiv \underset{e^{i} \geq 0}{\arg \max } E\left[v\left(\bar{w} h\left(e_{t}^{i}, \tilde{a}\right)+\bar{R}\left(b_{t}^{i}-e_{t}^{i}\right)\right)\right] .
$$

\footnotetext{
${ }^{18}$ Note that $A^{\prime}(I)<0$ if and only if $-v^{\prime \prime \prime}(I) / v^{\prime \prime}(I)>A(I)$, i.e., $-v^{\prime}(I)$ is "more concave" that $v(I)$. See, e.g., Gollier (2001) for a discussion of the plausibility of this assumption. See also Carroll (2002) for empirical evidence which is consistent with decreasing absolute risk aversion in the context of portfolio decisions in financial markets.
} 
One can then derive the following result.

Proposition 1. (Human capital investment). Under A1 and A3, the human capital investment is strictly increasing in family wealth, i.e., $e^{\prime}\left(b_{t}^{i}\right)>0$.

Proof. See appendix A.

Proposition 1 coincides with a result (derived from a two-period model with exogenous wealth) of the pioneering work by Levhari and Weiss (1974). (See also Eaton and Rosen, 1980.) The intuition for the result is the following. Under convention $h_{a}>0$, the assumption $h_{e a}>0$ implies that risk is increasing with the level of investment in human capital. In contrast, investing in physical capital (i.e., financial assets) is risk-free. Hence, if the degree of absolute risk aversion, $A(I)$, is decreasing in income $I$, such that $A\left(I_{t+1}^{i}\right)=A\left(I\left(b_{t}^{i}, \cdot, \cdot\right)\right)$ is decreasing in $b_{t}^{i}$, then individuals with larger $b_{t}^{i}$ invest more in risky education.

In fact, $e^{\prime}\left(b_{t}^{i}\right)>0$ is well-supported empirically even in advanced economies in which credit constraints seem to play a negligible role for human capital investments. ${ }^{19}$ (See section 5.3 for a brief review of empirical evidence.) There is no theoretical prediction, however, how the marginal propensity to invest in education, $e^{\prime}\left(b_{t}^{i}\right)$, changes with $b_{t}^{i}$. Moreover, the rare existing empirical evidence on the sign of $e^{\prime \prime}(b)$ is rather inconclusive. ${ }^{20}$ In order to focus the analysis, I shall thus suppose the following.

A4. The impact of a change in family wealth $b_{t}^{i}$ on the magnitude of the marginal propensity to invest in education is negligible, i.e., $\left|e^{\prime \prime}\left(b_{t}^{i}\right)\right| \approx 0$ for all $b_{t}^{i} \in \mathbb{R}_{+}$.

\footnotetext{
${ }^{19}$ Note that without uncertainty, i.e., if $\underline{a}=\bar{a} \equiv a$, the optimal schooling investment is given by $W h_{e}\left(e_{t}^{i}, a\right)=R$, according to (9). Thus, under certainty, $e_{t}^{i}$ is independent of $b_{t}^{i}$. For instance, this coincides with a result by Galor and Moav (2004) when credit constraints are not binding in their model.

${ }^{20}$ There exist some estimates for the impact of parental income on children's earnings which allow for non-linearity. Whereas Becker and Tomes (1986) suggest that the marginal impact is diminishing, if anything, Behrman and Taubman (1990) find a positive marginal impact. To see how these findings relate to the present model, first, define earnings of a member $i$ of generation $t$ as function of her parent's income (suppressing $\tilde{a}): E\left(I_{t}^{i}\right) \equiv \bar{w} h\left(e\left(b\left(I_{t}^{i}\right)\right)\right.$, $)$, where the relationships $e_{t}^{i}=e\left(b_{t}^{i}\right)$ and $b_{t}^{i}=b\left(I_{t}^{i}\right)$ have been used. Thus, $E^{\prime}(I)=\bar{w} h_{e}(e(b(I)), \cdot) e^{\prime}(b(I)) b^{\prime}(I)$ and $E^{\prime \prime}(I)=\bar{w}\left[h_{e e} e^{\prime}(b)^{2} b^{\prime}(I)^{2}+h_{e} e^{\prime \prime}(b) b^{\prime}(I)^{2}+h_{e} e^{\prime}(b) b^{\prime \prime}(I)\right]$. Thus, for instance, $E^{\prime \prime}(I) \approx 0$ if $e^{\prime \prime}(b) \approx 0$ and the effects driven $h_{e e}<0$ and $b^{\prime \prime}(I)>0$ (which is the empirically relevant case as argued in section 5.2) approximately cancel.
} 
Assumption A4 is not crucial for any of the mechanisms discussed in the subsequent analysis. However, it helps to focus the discussion on the role of diminishing marginal returns to human capital and the effects arising from intergenerational wealth transmission for the inequality-growth relationship.

\section{The Role of Inequality for Income Dynamics}

This section studies the aggregate behavior of the economy which results from individual decisions analyzed in the preceding section. In particular, it is examined how inequality in initial family wealth, for a given initial aggregate transfer $B_{0}$ (and conditional on initial GDP, $\left.Y_{0}=H_{0} f(\bar{k})\right)$, affects the process of development. For this purpose, it is useful to recall the dynamical system of the considered economy.

A given transfer $b_{t}^{i}$ to a member $i$ of generation $t$ is optimally allocated to savings, $s_{t}^{i}$, and education investments, $e_{t}^{i}=e\left(b_{t}^{i}\right)$, in period $t$ (Proposition 1). According to (2), this leads to an individual amount

$$
h_{t+1}^{i}=h\left(e\left(b_{t}^{i}\right), \tilde{a}\right) \equiv \hat{h}\left(b_{t}^{i}, \tilde{a}\right)
$$

of efficiency units of human capital, supplied during adulthood (which is a random variable). Thus, denoting the economy's c.d.f. of family wealth in period $t$ by $\Psi_{t}(b)$, with support $\mathcal{B}_{t} \subset \mathbb{R}_{+},{ }^{21}$ the aggregate human capital stock at $t+1$ is given by

$$
H_{t+1}=\int_{\mathcal{A}} \int_{\mathcal{B}_{t}} \hat{h}(b, \tilde{a}) d \Psi_{t}(b) d \Phi(\tilde{a})
$$

Aggregate income is given by $Y_{t+1}=H_{t+1} f(\bar{k}) \cdot{ }^{22}$

\footnotetext{
${ }^{21}$ Note from the assumptions on initial conditions that $\mathcal{B}_{0}=\left\{b_{0}^{P}, b_{0}^{R}\right\}$ and $\Psi_{0}(b)=0$ for $0 \leq b<b_{0}^{P}$, $\Psi_{0}(b)=1-\lambda$ for $b_{0}^{P} \leq b<b_{0}^{R}$, and $\Psi_{0}(b)=1$ for $b \geq b_{0}^{R}$.

${ }^{22}$ Note that the human capital risk considered in the model is consistent with risk associated with skill specificity in the following sense. Suppose individuals acquire skills which are applicable in a single "industry" only (which may also be interpreted as specific task) and there are ideosyncratic productivity shocks across industries (Wildasin, 2000). To see that this is consistent with the risk considered here, suppose there is a continuum $[0,1]$ of intermediate goods industries, indexed by $j$. Output $q_{t}(j)$ in industry $j$ at $t$ is produced with industry-specific human capital $\hat{H}_{t}(j) \equiv \int_{\mathcal{B}_{t}} \hat{h}(b, \theta(j)) d \Psi_{t}(b)$,
} 
According to (3) and (10), given realization $a$ of the random variable $\tilde{a}$ after educational investments are made, income in $t+1$ of an adult individual $i$ reads

$$
I_{t+1}^{i}=\bar{w} h\left(e\left(b_{t}^{i}\right), a\right)+\bar{R}\left(b_{t}^{i}-e\left(b_{t}^{i}\right)\right)=I\left(b_{t}^{i}, e\left(b_{t}^{i}\right), a\right) \equiv \hat{I}\left(b_{t}^{i}, a\right)
$$

$I_{t+1}^{i}$ is then optimally allocated to consumption, $c_{t+1}^{i}$, and transfers to the offspring,

$$
b_{t+1}^{i}=b\left(I_{t+1}^{i}\right)=b\left(\hat{I}\left(b_{t}^{i}, a\right)\right) \equiv \hat{b}\left(b_{t}^{i}, a\right)
$$

According to (13), the wealth transfer within each dynasty $i$ follows a discrete time Markov process defined by the difference equation $b_{t+1}^{i}=\hat{b}\left(b_{t}^{i}, \tilde{a}\right)$.

Due to the small open economy assumption, there are no feedback effects through factor price changes from aggregate variables to individual behavior. This not only simplifies the analysis but also excludes the mechanism suggested by the classical view: i.e., that higher inequality fosters growth fueled by domestic accumulation of physical capital if the marginal propensity to save is increasing. It will turn out, however, that wealth accumulation nevertheless plays a fundamental role for the inequality-growth relationship by affecting resources available for human capital accumulation at the individual level.

For simplicity, let me restrict attention to the case in which even for the worst realizations of $\tilde{a}$, income of an adult increases (without bound) with the amount of transfer received as child. ${ }^{23}$ Formally, this means the following.

where where $\theta(j)$ is the realization of an i.i.d. shock $\tilde{\theta}$. (Note that because industries are symmetric and $b$ and $\tilde{\theta}$ are independently distributed, skill supply across industries is fully symmetric.) Suppose this production technology simply reads $q_{t}(j)=\hat{H}_{t}(j)$. Thus, the the aggregate stock of human capital in period $t+1, H_{t+1}=\int_{j \in[0,1]} \hat{H}_{t}(j) d j=\int_{j \in[0,1]} q_{t}(j) d j$ can be thought of a composite input of (perfectly substitutable) intermediate goods. Let $\tilde{\theta}$ and $\tilde{a} \in \mathcal{A}$ (still with c.d.f. $\Phi(\tilde{a})$ ) be related such that $\theta(j) \equiv \Phi^{-1}(j)$ is the realization of the shock in industry $j \in[0,1]$ (i.e., after realization of shocks, industries are ordered such that $j=\Phi(a)$, where $a$ is a realization of $\tilde{a})$. Thus, one can write $H_{t+1}=\int_{j \in[0,1]} \int_{\mathcal{B}_{t}} \hat{h}(b, \tilde{\theta}(j)) d \Psi_{t}(b) d j=\int_{\mathcal{A}} \int_{\mathcal{B}_{t}} \hat{h}(b, \tilde{a}) d \Psi_{t}(b) d \Phi(\tilde{a})$, which coincides with (11).

${ }^{23}$ Moreover, it is implicitly assumed throughout the paper that any young individual with zero wealth is able to pay back the loan $\bar{R} e(0)$, which equals such an individual's optimal amount of lending, by her labor income even for the worst realization of the shock $\underline{a}$, i.e., $\hat{I}(0, \underline{a})=\bar{w} h(e(0), \underline{a})-\bar{R} e(0) \geq 0$. 
A5. For all $a \in \mathcal{A}, \hat{I}_{b}(b, a)>0, b \in \mathbb{R}_{+}$, and $\lim _{b \rightarrow \infty} \hat{I}(b, a)=\infty .^{24}$

The next two results characterize the Markov process $b_{t+1}^{i}=\hat{b}\left(b_{t}^{i}, \tilde{a}\right)$.

Lemma 3. Under A2 and A5, for all $a \in \mathcal{A}$. There exists $\underline{b}_{a} \geq 0$ such that $\hat{b}(b, a)>0$ and $\hat{b}_{b}(b, a)>0$ for all $b>\underline{b}_{a}$.

Proof. Recall from Lemma 1 (which is implied by A2) that there exists $\underline{I} \geq 0$ such that $b(I)>0$ and $b^{\prime}(I)>0$ if $I>\underline{I}$. Also recall from $(13)$ that $\hat{b}(b, a)=b(\hat{I}(b, a))$, which implies $\hat{b}_{b}(b, a)=b^{\prime}(\hat{I}(b, a)) \hat{I}_{b}(b, a)$. Thus, under A5, for all $a \in \mathcal{A}$, a gradual increase in $b$ eventually must lead to a level of $b$, denoted by $\underline{b}_{a}$, such that $\hat{b}(b, a)>0$ and $\hat{b}_{b}(b, a)>0$ for all $b>\underline{b}_{a}$. This concludes the proof.

For notational simplicity in what follows, suppress the random variable $\tilde{a}$ by defining wealth and income one period after (indicated by subscript ' 1 ') the level of wealth started in $b_{t}^{i}$ as

$$
\begin{aligned}
\tilde{b}_{1}\left(b_{t}^{i}\right) & \equiv \hat{b}\left(b_{t}^{i}, \tilde{a}\right), \\
\tilde{I}_{1}\left(b_{t}^{i}\right) & \equiv \hat{I}\left(b_{t}^{i}, \tilde{a}\right) .
\end{aligned}
$$

Lemma 4. Under A1-A5, for any realization $a \in \mathcal{A}$ of the random shock $\tilde{a}$. (i) If $b^{\prime \prime}(I) \leq 0$, then $\tilde{b}_{1}^{\prime \prime}(b) \leq 0$. (ii) If, by contrast, $b^{\prime \prime}(I)>0$, then the sign of $\tilde{b}_{1}^{\prime \prime}(b)$ is ambiguous. ${ }^{25}$

Proof. First, note that (whenever differentiable)

$$
\tilde{b}_{1}^{\prime}(b)=b^{\prime}\left(\tilde{I}_{1}(b)\right) \tilde{I}_{1}^{\prime}(b),
$$

\footnotetext{
${ }^{24}$ Using (12), it is easy to check that $e^{\prime}(b) \leq 1$ is sufficient for $\hat{I}_{b}(b, a)>0$ to hold. That is, if a marginal increase in $b$ does not lead to a decline of investment in the financial market, then $\hat{I}_{b}>0$. However, although plausible, $e^{\prime}(b) \leq 1$ is not ensured by the assumptions made so far. One can show, for instance, that $e^{\prime}(b)<1$ if, for all $a \in \mathcal{A},-\frac{A^{\prime}(\hat{I}(b, a)) \bar{w}}{A(\hat{I}(b, a))} \geq \frac{h_{e \tilde{a}}(e(b), a)}{h_{\tilde{a}}(e(b), a) h_{e}(e(b), a)}$, or if $\left|h_{e e}\right|$ is sufficiently large.

${ }^{25}$ Note that in the case $b(I)=0$ for $I \leq \underline{I}$ and $b(I)>0$ for $I>\underline{I}, \underline{I} \geq 0$, the function $b(I)$ is not differentiable at $I=\underline{I}$ (and $b^{\prime}(I)>0$ for $I>\underline{I}$ under A2, according to Lemma 1). The subsequent analysis neglects this for simplicity, implicitly stating results for $I \neq \underline{I}$ only.
} 
according to (13)-(15). Hence,

$$
\tilde{b}_{1}^{\prime \prime}(b)=b^{\prime \prime}\left(\tilde{I}_{1}(b)\right) \tilde{I}_{1}^{\prime}(b)^{2}+b^{\prime}\left(\tilde{I}_{1}(b)\right) \tilde{I}_{1}^{\prime \prime}(b)
$$

Moreover, according to (12) and (15), we have

$$
\begin{aligned}
& \tilde{I}_{1}^{\prime}(b)=\left(\bar{w} h_{e}(e(b), a)-\bar{R}\right) e^{\prime}(b)+\bar{R}, \\
& \tilde{I}_{1}^{\prime \prime}(b)=\bar{w} h_{e e}(e(b), a) e^{\prime}(b)^{2}+\left(\bar{w} h_{e}(e(b), a)-\bar{R}\right) e^{\prime \prime}(b),
\end{aligned}
$$

$a \in \mathcal{A}$. Under A4, we can neglect the second summand on the right-hand side of (19), i.e., $\tilde{I}_{1}^{\prime \prime}(b)<0$, according to $h_{e e}<0$ and $e^{\prime}(b)>0$ (Proposition 1 ) under A1 and A3. Hence, under A2 (which implies $b^{\prime}(I) \geq 0$, according to Lemma 1 ), we have $\tilde{b}_{1}^{\prime \prime}(b) \leq 0$ if $b^{\prime \prime}(I) \leq 0$, according to (17). This confirms part (i). To confirm part (ii), note that, if $b^{\prime \prime}(I)>0$, the sign of the first summand on the right-hand side of (17) is strictly positive for all $a \in \mathcal{A}$ under A5. This concludes the proof.

According to Lemma 4, if the saving function $b(I)$ is concave (i.e., if $b^{\prime \prime}(I) \leq 0$ ), then the transfer of an adult to her offspring will be concave in the transfer she received as a child (i.e., $\tilde{b}_{1}^{\prime \prime}(b) \leq 0$ ). However, if the marginal propensity to save (for adults) is increasing (i.e., if $b^{\prime \prime}(I)>0$ ), the transfer of an adult to her offspring may be convex in the transfer received herself as a child (i.e., $\tilde{b}_{1}^{\prime \prime}(b)>0$ is possible). The empirical relevance of an increasing marginal propensity to save (and bequeath, respectively) is discussed in section 5.2. Its consequences for the process of development are analyzed in the following.

\subsection{Inequality and the Process of Development}

Recall that, initially, there are two groups of individuals, rich and poor, and the aggregate initial transfer is $B_{0}=\lambda b_{0}^{R}+(1-\lambda) b_{0}^{P}$. To study the role of inequality for the growth process, suppose the distribution of initial transfers changes (in a lump-sum 
fashion) to

$$
\breve{b}_{0}^{R} \equiv b_{0}^{R}-\varepsilon, \quad \breve{b}_{0}^{P} \equiv b_{0}^{P}+\varepsilon \lambda /(1-\lambda)
$$

i.e., aggregate family wealth, $B_{0}$, is held constant. Under restriction $\varepsilon<(1-\lambda)\left(b_{0}^{R}-b_{0}^{P}\right)$, i.e., as long as $\breve{b}_{0}^{P}<\breve{b}_{0}^{R}$, the economy is said to be more equal, the higher $\varepsilon$. The following analysis derives comparative-static results with respect to changes in $\varepsilon .{ }^{26}$

\subsubsection{Short Run Impact of Higher Equality}

Let $g_{s, t} \equiv\left(Y_{s}-Y_{t}\right) / Y_{t}$ be the growth rate of aggregate output (or GDP, respectively) between periods $s$ and $t, s>t \geq 0$. First, consider the impact of an increase in $\varepsilon$ on the aggregate human capital stock $H_{1}$, which gives us the short run effect of higher equality on aggregate (or per capita) income, $Y_{1}=H_{1} f(\bar{k})$, and thus, on the initial growth rate $g_{1,0}=H_{1} / H_{0}-1$ of the economy (recall $Y_{0}=H_{0} f(\bar{k})$ ). Again, suppressing the random variable for notational simplicity, let

$$
\tilde{h}_{1}\left(b_{t}^{i}\right) \equiv \hat{h}\left(b_{t}^{i}, \tilde{a}\right)
$$

Under (20), the aggregate human capital stock in period $1, H_{1}$, can be written as

$$
\begin{aligned}
H_{1} & =E\left[\lambda \tilde{h}_{1}\left(\breve{b}_{0}^{R}\right)+(1-\lambda) \tilde{h}_{1}\left(\breve{b}_{0}^{P}\right)\right] \\
& =E\left[\lambda \tilde{h}_{1}\left(b_{0}^{R}-\varepsilon\right)+(1-\lambda) \tilde{h}_{1}\left(b_{0}^{P}+\varepsilon \lambda /(1-\lambda)\right)\right] \equiv \hat{H}_{1}(\varepsilon),
\end{aligned}
$$

according to (11) and the specification of the initial distribution of transfers. From this, we obtain the following result.

Proposition 2. (Impact of higher equality in the short run). Under A1, A3 and A4, higher equality of initial family wealth is associated with higher aggregate income $Y_{1}$ (and thus, faster growth $g_{1,0}$ ), i.e., $\hat{H}_{1}^{\prime}(\varepsilon)>0$.

\footnotetext{
${ }^{26}$ Note that income inequality and inequality of family wealth are closely related, according to Lemma 1.
} 
Proof. According to $(22)$, differentiating $\hat{H}_{1}(\varepsilon)$ with respect to $\varepsilon$ yields

$$
\begin{aligned}
\hat{H}_{1}^{\prime}(\varepsilon) & =\lambda E\left[\tilde{h}_{1}^{\prime}\left(\breve{b}_{0}^{P}\right)-\tilde{h}_{1}^{\prime}\left(\breve{b}_{0}^{R}\right)\right], \text { where } \\
\tilde{h}_{1}^{\prime}(b) & =h_{e}(e(b), a) e^{\prime}(b),
\end{aligned}
$$

$a \in \mathcal{A}$, according to (10) and (21). Since $\breve{b}_{0}^{P}<\breve{b}_{0}^{R},(23)$ implies that $\hat{H}_{1}^{\prime}(\varepsilon)>0$ if, for instance, for all $a \in \mathcal{A}$ and for all $b \in \mathbb{R}_{++}, \tilde{h}_{1}^{\prime \prime}(b)<0$. Note that

$$
\tilde{h}_{1}^{\prime \prime}(b)=h_{e e}(e(b), a) e^{\prime}(b)^{2}+h_{e}(e(b), a) e^{\prime \prime}(b),
$$

according to (24). The first summand on the right-hand side of (25) is negative since $e^{\prime}(b)>0$, according to Proposition 1 (which holds under A1 and A3), and $h_{e e}<0$. Thus, if $e^{\prime \prime}(b) \leq 0$, or if $e^{\prime \prime}(b)$ is positive but small in magnitude as supposed in A4, we have $\tilde{h}_{1}^{\prime \prime}(b)<0$. This confirms the result.

Proposition 2 is in line with a standard result in the literature on inequality and growth when growth is driven by human capital investments (e.g., Galor and Zeira, 1993; Bénabou, 1996; Moav, 2002). Intuitively, higher equality fosters growth when the poor have a higher expected marginal return to education. This applies when two conditions are met. First, individual human capital investment is an increasing function of wealth (usually derived from borrowing constraints rather than from uncertainty as in the present paper), which holds since $e^{\prime}(\cdot)>0$, according to Proposition 1. Second, marginal returns to education are diminishing, which is reflected by the assumption $h_{e e}<0$ in A1. Hence, the aggregate human capital stock typically increases in the short run if (initial) wealth, and thus, if (initial) educational investment is spread over the population more equally. However, as will become apparent in the following, due to the effects of intergenerational wealth transmission, this may not hold anymore in the medium run. 


\subsubsection{Medium Run Impact of Higher Equality}

Given family wealth $b_{t}^{i}$ in period $t$, human capital of a member $i$ of generation $t+1$ in period $t+2$ is

$$
h_{t+2}^{i}=\hat{h}\left(b_{t+1}^{i}, \tilde{a}\right)=\tilde{h}_{1}\left(b_{t+1}^{i}\right)=\tilde{h}_{1}\left(\hat{b}\left(b_{t}^{i}, \tilde{a}\right)\right)=\tilde{h}_{1}\left(\tilde{b}_{1}\left(b_{t}^{i}\right)\right) \equiv \tilde{h}_{2}\left(b_{t}^{i}\right),
$$

according to (10), (21), (13) and (14). For later use, the following auxiliary results are established.

Lemma 5. Under A1-A3 and A5, for any realizations of random shocks, $\tilde{h}_{2}^{\prime}(\cdot) \geq 0$.

Proof. First, note that

$$
\tilde{h}_{2}^{\prime}(b)=\tilde{h}_{1}^{\prime}\left(\tilde{b}_{1}(b)\right) \tilde{b}_{1}^{\prime}(b)=h_{e}\left(e\left(\tilde{b}_{1}(b)\right), \tilde{a}\right) e^{\prime}\left(\tilde{b}_{1}(b)\right) \tilde{b}_{1}^{\prime}(b)
$$

according to (10), (21) and (26). Substituting (16) into (27) leads to

$$
\tilde{h}_{2}^{\prime}(b)=h_{e}\left(e\left(\tilde{b}_{1}(b)\right), \tilde{a}\right) e^{\prime}\left(\tilde{b}_{1}(b)\right) b^{\prime}\left(\tilde{I}_{1}(b)\right) \tilde{I}_{1}^{\prime}(b) .
$$

Under A2, (whenever differentiable) $b^{\prime}(\cdot) \geq 0$, according to Lemma 1, and, under A1 and $\mathrm{A} 3, e^{\prime}(\cdot)>0$, according to Proposition 1. Moreover, $\tilde{I}_{1}^{\prime}(b)>0$ under A5. Thus, recalling $h_{e}>0$, Lemma 5 follows from (28).

Lemma 6. Under A1-A5, for any realizations of random shocks. If $b^{\prime \prime}(I) \leq 0$, then $\tilde{h}_{2}^{\prime \prime}(b) \leq 0$. (ii) If, by contrast, $b^{\prime \prime}(I)>0$, then the sign of $\tilde{h}_{2}^{\prime \prime}(b)$ is ambiguous.

Proof. Note that (27) implies

$$
\tilde{h}_{2}^{\prime \prime}(b)=h_{e e}\left(e\left(\tilde{b}_{1}(b)\right), \tilde{a}\right) e^{\prime}\left(\tilde{b}_{1}(b)\right)^{2} \tilde{b}_{1}^{\prime}(b)^{2}+h_{e}\left(e\left(\tilde{b}_{1}(b)\right), \tilde{a}\right)\left[e^{\prime \prime}\left(\tilde{b}_{1}(b)\right) \tilde{b}_{1}^{\prime}(b)^{2}+e^{\prime}\left(\tilde{b}_{1}(b)\right) \tilde{b}_{1}^{\prime \prime}(b)\right] .
$$

The first summand on the right-hand side of (29) is non-positive since $h_{e e}<0$. Moreover, if $\left|e^{\prime \prime}(b)\right|$ is small in magnitude as supposed in $\mathrm{A} 4$, the first term in square brackets of (29) is negligible. Regarding the second term in square brackets, if $b^{\prime \prime}(I) \leq 0$, then 
$\tilde{b}_{1}^{\prime \prime}(\cdot) \leq 0$ according to part (i) of Lemma 4 . In addition, recall $e^{\prime}(\cdot)>0$ from Proposition 1 (which holds under A1 and A3). Thus, if $b^{\prime \prime}(I) \leq 0$, then the second term in square brackets of (29) is non-positive. Hence, $\tilde{h}_{2}^{\prime \prime}(b) \leq 0$ if $b^{\prime \prime}(I) \leq 0$, confirming part (i) of Lemma 6 . However, if $b^{\prime \prime}(I)>0$, then we may have $\tilde{b}_{1}^{\prime \prime}(b)>0$, according to part (ii) of Lemma 4. Consequently, we may have $\tilde{h}_{2}^{\prime \prime}(b)>0$ if $b^{\prime \prime}(I)>0$, confirming part (ii) of Lemma 6. This concludes the proof.

Given $\breve{b}_{0}^{i}$, the intergenerational transfer within dynasty $i$ in period 1, after realization $a_{0} \in \mathcal{A}$ of the shock at $t=0$, is given by $\breve{b}_{1}^{i} \equiv \hat{b}\left(\breve{b}_{0}^{i}, a_{0}\right), i=R, P$. Thus, using (20) and (26), the aggregate human capital stock in period 2 may be written as

$$
\begin{aligned}
H_{2} & =E\left[\lambda E\left[\tilde{h}_{1}\left(\breve{b}_{1}^{R}\right)\right]+(1-\lambda) E\left[\tilde{h}_{1}\left(\breve{b}_{1}^{P}\right)\right]\right] \\
& =E\left[\lambda E\left[\tilde{h}_{2}\left(b_{0}^{R}-\varepsilon\right)\right]+(1-\lambda) E\left[\tilde{h}_{2}\left(b_{0}^{P}+\varepsilon \lambda /(1-\lambda)\right)\right]\right] \equiv \hat{H}_{2}(\varepsilon) .
\end{aligned}
$$

This leads to the following result.

Lemma 7. Under A1-A5, for any realizations of random shocks. If $b^{\prime \prime}(I) \leq 0$, then $\hat{H}_{2}^{\prime}(\varepsilon) \geq 0$. (ii) If, by contrast, $b^{\prime \prime}(I)>0$, then we may have $\hat{H}_{2}^{\prime}(\varepsilon)<0$.

Proof. First, note that (30) implies

$$
\hat{H}_{2}^{\prime}(\varepsilon)=\lambda E\left[E\left[\tilde{h}_{2}^{\prime}\left(\breve{b}_{0}^{P}\right)-\tilde{h}_{2}^{\prime}\left(\breve{b}_{0}^{R}\right)\right]\right]
$$

Since $\breve{b}_{0}^{P}<\breve{b}_{0}^{R}$, (31) implies that $\hat{H}_{2}^{\prime}(\varepsilon)>(=,<) 0$ if, for instance, for all realizations $a_{0}, a_{1} \in \mathcal{A}$ at $t=0,1$ and for all $b \in \mathbb{R}_{+}, \tilde{h}_{2}^{\prime \prime}(b)<(=,>) 0$. Finally, use Lemma 6 . This confirms Lemma 7.

The intuition of Lemma 7 is as follows. On the one hand, a diminishing marginal return to human capital investment leads to a positive impact of higher equality of family wealth on $\mathrm{H}_{2}$, i.e., the short run effect of higher equality on the aggregate human capital stock which underlies Proposition 2 also applies two periods after a lump-sum redistribution. To see this intuitively, recall that the wealth transfer from parent to 
child is an increasing function of parent's income. Consequently, an increase in $\varepsilon$ implies that members of generation 0 which belong to a poor dynasty (endowed with $b_{0}^{P}$ ) are, on average, richer as parents, and thus transfer, on average, more to their offspring (i.e., to members of generation 1). In turn, on average, members of generation 1 from poor families invest more in education whereas the opposite holds for rich dynasties (with family wealth $b_{0}^{R}$ in the initial period). Hence, on the other hand, and in contrast to the short run effect of higher equality, the impact of an increase in $\varepsilon$ on the aggregate human capital stock in period $2, \mathrm{H}_{2}$, also depends on the intergenerational wealth transmission as function of parents' income. As a consequence, the positive effect from $h_{e e}<0$ on aggregate human capital investment may be dominated: Poor dynasties transmit more wealth, on average, when $\varepsilon$ is higher, rich dynasties transmit less. If $b^{\prime \prime}(I)>0$, then the average reduction in wealth transmission of the rich may outweigh the average increase in wealth transmission of the poor. Formally, this has been established in Lemma 4, which states that $\tilde{b}_{1}^{\prime \prime}(b)>0$ is possible if $b^{\prime \prime}(I)>0$. As implied by Lemma 6 , initially richer dynasties may thus sharply reduce their educational investment, on average. Thus, despite diminishing marginal returns to education, reflected by $h_{e e}<0, H_{2}=$ $\hat{H}_{2}(\varepsilon)$ may decrease with $\varepsilon$. Intuitively, the "more convex" $b(I)$ and the "less concave" $h(e, \cdot)$ in $e$ is, the "more likely" is $\hat{H}_{2}^{\prime}(\varepsilon)<0$, which reflects a positive relationship between inequality and growth.

To draw conclusions for the role of (initial) inequality for the process of development in the medium run, i.e., to examine the impact of an increase in $\varepsilon$ on the growth rate $g_{t, 0}\left(=Y_{t} / Y_{0}-1=H_{t} / H_{0}-1\right)$ for $t \geq 2$, let

$$
h_{t+3}^{i}=\tilde{h}_{2}\left(b_{t+1}^{i}\right)=\tilde{h}_{2}\left(\tilde{b}_{1}\left(b_{t}^{i}\right)\right) \equiv \tilde{h}_{3}\left(b_{t}^{i}\right)
$$

where (26) has been used. Thus, recalling (20), the aggregate human capital stock in period 3 may be written as

$$
H_{3}=E\left[E\left[\lambda E\left[\tilde{h}_{3}\left(b_{0}^{R}-\varepsilon\right)\right]+(1-\lambda) E\left[\tilde{h}_{3}\left(b_{0}^{P}+\varepsilon \lambda /(1-\lambda)\right)\right]\right]\right] \equiv \hat{H}_{3}(\varepsilon)
$$


As can be seen from comparing (33) with (30), the structure of the development process through wealth transmission and human capital investments remains similar from period 2 onwards. In fact, in line with Lemma 7, the following can be concluded.

Proposition 3. (Impact of higher equality in the medium run). Under A1-A5, for all finite $t \geq 2$. (i) If $b^{\prime \prime}(I) \leq 0$, higher equality of initial family wealth has a positive impact on the subsequent growth rate $g_{t, 0}$. (ii) If, however, $b^{\prime \prime}(I)>0$, then the relationship between equality and $g_{t, 0}$ may be negative.

Proof. Note that (33) implies

$$
\begin{aligned}
\hat{H}_{3}^{\prime}(\varepsilon) & =\lambda E\left[E\left[E\left[\tilde{h}_{3}^{\prime}\left(\breve{b}_{0}^{P}\right)+\tilde{h}_{3}^{\prime}\left(\breve{b}_{0}^{R}\right)\right]\right]\right], \text { where } \\
\tilde{h}_{3}^{\prime}(b) & =\tilde{h}_{2}^{\prime}\left(\tilde{b}_{1}(b)\right) \tilde{b}_{1}^{\prime}(b),
\end{aligned}
$$

according to the definition $\tilde{h}_{3}(b)=\tilde{h}_{2}\left(\tilde{b}_{1}(b)\right)$ in (32). Since $\breve{b}_{0}^{P}<\breve{b}_{0}^{R}$, (34) implies that $\hat{H}_{3}^{\prime}(\varepsilon)>(=,<) 0$ if, for instance, for all realizations $a_{0}, a_{1}, a_{2} \in \mathcal{A}$ at $t=0,1,2$, and for all $b \in \mathbb{R}_{+}, \tilde{h}_{3}^{\prime \prime}(b)<(=,>) 0$. Also note that (35) implies

$$
\tilde{h}_{3}^{\prime \prime}(b)=\tilde{h}_{2}^{\prime \prime}\left(\tilde{b}_{1}(b)\right) \tilde{b}_{1}^{\prime}(b)^{2}+\tilde{h}_{2}^{\prime}\left(\tilde{b}_{1}(b)\right) \tilde{b}_{1}^{\prime \prime}(b)
$$

Recall that $\tilde{h}_{2}^{\prime}(\cdot) \geq 0$, according to Lemma 5. First, suppose $b^{\prime \prime}(I) \leq 0$. Thus, $\tilde{h}_{3}^{\prime \prime}(b) \leq$ 0 , since $\tilde{b}_{1}^{\prime \prime}(\cdot) \leq 0$ and $\tilde{h}_{2}^{\prime \prime}(b) \leq 0$ in this case, according to part (i) of Lemma 4 and part (i) of Lemma 6, respectively. Using (36), this confirms part (i) of Proposition 3. In an analogous fashion, part (ii) of Proposition 3 follows from (36) together with part (ii) of Lemma 4 and part (ii) of Lemma 6 . Thus, the impact of an increase in $\varepsilon$ on $H_{3}$ is similar to its impact on $H_{2}$, where the latter has been established in Lemma 7. The impact of an increase in $\varepsilon$ on $H_{4}$ and higher can be established in a completely analogous fashion, employing a very similar structure, which yields similar results. This concludes the proof.

Proposition 3 shows that the medium run impact of higher equality on human capital accumulation and growth crucially depends on the properties of the saving 
function. Whether part (i) or part (ii) of Proposition 3 is the relevant case is thus an empirical question, which is addressed in section 5.2.

\subsection{Inequality and Aggregate Income in Stationary Equilib- rium}

What is the impact of initial inequality on the aggregate human capital stock and per capita income in the long run (i.e., as $t \rightarrow \infty$ ), denoted by $H_{\infty}$ and $Y_{\infty}=H_{\infty} f(\bar{k})$, respectively? Answering this question requires an analysis of the long run behavior of wealth transfers within dynasties, which are governed by the Markov process $b_{t+1}^{i}=$ $\hat{b}\left(b_{t}^{i}, \tilde{a}\right)$, defined by (13). Due to the uncertainty in the model, these transfers never reach steady state points as known from deterministic models. Therefore, the goal is to find stationary equilibria in the sense that, as $t \rightarrow \infty$, the distribution of $b_{t}^{i}$ within dynasty $i$ is time-invariant. The following discussion of such stationary equilibria, and its consequences for the relationship between inequality and per capita income in the long run deals with simple cases in a rather informal way. Both a more formal and more general treatment is provided in appendix B.

To focus the discussion on empirically plausible situations, suppose intergenerational transfers are zero for low levels of income. ${ }^{27}$ Moreover, to prevent infinite wealth accumulation of rich dynasties, suppose that for high wealth levels $b^{i}$ and for $a=\underline{a}, \bar{a}$, $\hat{b}\left(b^{i}, a\right)$ is strictly concave as function of $b^{i}{ }^{28}$ Simple cases which meet these two criteria are depicted in Fig. 1. ${ }^{29}$ Appendix B shows that the main conclusions derived from Fig. 1 remain valid more generally.

\section{$<$ Figure 1 $>$}

\footnotetext{
${ }^{27}$ For the US, for instance, it has been frequently confirmed that the mean savings rate of households in the lowest quintile of the income distribution is non-positive (e.g., Browning and Lusardi, 1996; Dynan et al., 2000).

${ }^{28}$ Note that according to $(17)$ and $(19)$, even if $b^{\prime \prime}(I)>0$, for high $I$, this occurs under weak conditions since $h_{e e}<0$.

${ }^{29}$ Note that for $b_{t+1}^{i}>0$, the $\hat{b}$-curves for $\underline{a}$ and $\bar{a}$ do not intersect because $\hat{b}_{a}(b, a)>0$ in this case. Also note that, under A2 and A5, $\hat{b}\left(b^{i}, a\right)$ is strictly increasing in $b^{i}$ for $a=\underline{a}, \bar{a}$ if $b^{i}>\underline{b} \underline{a}, \underline{b}_{\bar{a}}$, according to Lemma 3.
} 
Panel (a) shows a situation in which, irrespective of initial wealth holdings, for any dynasty $i, b_{t}^{i}=0$ as $t \rightarrow \infty$ with probability one. This is called a trivial stationary equilibrium. (In panel (a), this equilibrium is also globally stable.) Obviously, in this case, higher equality of initial wealth has no impact on per capita income in the long run, $Y_{\infty}$.

In panel (b), if $b_{0}^{i} \leq c_{\bar{a}}$, again, wealth levels within dynasty $i$ become zero with probability one in the long run (which now is a locally stable stationary equilibrium). If $b_{0}^{i} \geq c_{\underline{a}}$, then the distribution of $b_{t}^{i}$ converges with probability one to a locally unique stable stationary equilibrium on the interval $\left[d_{\underline{a}}, d_{\bar{a}}\right]$. Thus, if $b_{0}^{i} \leq c_{\bar{a}}$ or if $b_{0}^{i} \geq c_{\underline{a}}$ for $i=R, P$, there is no impact of higher equality on $Y_{\infty}$. If $b_{0}^{i} \in\left(c_{\bar{a}}, c_{\underline{a}}\right)$, then, as $t \rightarrow \infty$, both $b_{t}^{i}=0$ or $b_{t}^{i} \in\left[d_{\underline{a}}, d_{\bar{a}}\right]$ is possible with positive probability. ${ }^{30}$ Thus, according to the law of large numbers, if $b_{0}^{P} \in\left(c_{\bar{a}}, c_{\underline{a}}\right)$, some fraction $q \in(0,1)$ of initially poor dynasties will end up with zero wealth in the long run. Also by the law of large numbers, note that this fraction $q$ is increasing in the distance of $b_{0}^{P}$ to $c_{\underline{a}}$ (or decreasing in $\left(b_{0}^{P}-c_{\bar{a}}\right)$, respectively). Thus, the lower $\left(c_{\underline{a}}-b_{0}^{P}\right)$ is, the larger the fraction of initially poor dynasties which transmit positive wealth levels in the long run. Hence, if $b_{0}^{R}>c_{\underline{a}}$ and $b_{0}^{P} \in\left(c_{\bar{a}}, c_{\underline{a}}\right)$, then any redistribution to the poor which leaves wealth levels of initially rich individuals sufficiently high, ${ }^{31}$ unambiguously leads to higher average human capital investments, $H_{\infty}$, if $e^{\prime}(b)>0$ (as ensured under A1 and A3, according to Proposition 1), and thus, to a higher $Y_{\infty}$. This case may be relevant in advanced countries, i.e., if there is a large group of sufficiently rich individuals. Whereas in this situation there is a negative link between inequality and $Y_{\infty}$, there may be a positive link in other situations. To see this, suppose $b_{0}^{i} \in\left(0, c_{\bar{a}}\right]$ for $i=R, P$, such that the distribution of wealth levels of all dynasties converge with probability one to the trivial stationary equilibrium. ${ }^{32}$ In this case, sufficient redistribution to the rich

\footnotetext{
${ }^{30}$ For illuminating discussions of this stationary equilibrium indeterminacy in stochastic models, see, e.g., Laitner (1981) and Wang (1993).

${ }^{31}$ More precisely, this refers to any increase in $\varepsilon$ small enough such that $\breve{b}_{0}^{R}=b_{0}^{R}-\varepsilon \geq c_{\underline{a}}$, implying that the distribution of wealth holdings of initially rich dynasties still converges with probability one to a locally unique stationary equilibrium on the interval $\left[d_{a}, d_{\bar{a}}\right]$.

${ }^{32}$ This reflects a poverty trap of the kind often encountered in the literature on inequality and growth (which here is derived from a stochastic model).
} 
may result in a situation in which wealth levels of at least some initially rich dynasties converge to the stationary equilibrium on the interval $\left[d_{\underline{a}}, d_{\bar{a}}\right]$, without affecting the long run wealth distribution of the initially poor (who end up with zero wealth anyway). Thus, if $e^{\prime}(b)>0, Y_{\infty}$ is raised. Finally, if $b_{0}^{i} \in\left(c_{\bar{a}}, c_{\underline{a}}\right)$ for $i=R, P$, the impact of higher equality on $Y_{\infty}$ is ambiguous. In sum, we may conclude the following from this discussion.

Proposition 4. (Impact of higher equality in the long run). Suppose a stationary equilibrium of the Markov process $b_{t+1}^{i}=\hat{b}\left(b_{t}^{i}, \tilde{a}\right)$ exists. Then the relationship between equality and long run income, $Y_{\infty}$, is generally ambiguous, irrespective of the shape of the saving function, $b(I)$.

Thus, even if there is a systematic relationship between initial inequality and aggregate income in the short run and medium run (see Propositions 2 and 3 in section 4.1), one cannot draw general conclusions regarding the relationship between initial inequality and $Y_{\infty}$ from the shape of the saving function. However, whereas higher inequality may help to overcome a poverty trap in poor economies, if anything, the analysis suggests that the relationship between initial inequality and long run income is negative for advanced countries.

\section{Empirical Relevance}

This section reviews empirical evidence regarding crucial features and results of the theory developed in this paper.

\subsection{The Inequality-Growth Relation Revisited}

The present paper has suggested a channel through which initial inequality may have a positive impact on the growth rate of aggregate income in the medium run. To address this result in the light of empirical evidence, first, it should be noted that the usual modelling approach in growth empirics relies on some hypothesis of conditional 
convergence (e.g., Barro, 1991, 2000). That is, regression analysis regarding the determinants of economic growth, like inequality of income, usually control for the level of per capita income in some base year (properly instrumented) to account for the stage of development of an economy, like initial GDP, $Y_{0} .{ }^{33}$ This focus on the transition path enables us to compare the hypotheses derived in section 4.1 (particularly regarding the medium run) with empirical evidence in this literature.

The earlier literature has suggested a negative effect of income inequality on growth (e.g., Alesina and Rodrik, 1994; Persson and Tabillini, 1994; Perotti, 1996), which however, is not based on panels. Using a new and comprehensive high-quality data set, Deininger and Squire (1998) and Banerjee and Duflo (2003) find practically none, whereas Li and Zou (1998) and Forbes (2000) report a positive and significant medium run effect. ${ }^{34}$ The most interesting results for the present paper, however, emerge in a recent study of Barro (2000), who allows income inequality (measured by the Gini coefficient, using the same data set as Deininger and Squire, 1998) to interact with (the log of) per capita GDP in growth regressions. His evidence suggests a non-linear impact of inequality on GDP growth, with a negative relationship for less developed countries (with GDP per capita below around \$2000) and a positive relationship for more advanced countries (with GDP per capita above \$2000). ${ }^{35}$ From these results, Barro (2000, p.18) concludes that "for rich countries, where credit constraints are less serious, the growth-promoting aspects of inequality may dominate". To the best of my knowledge, the only available theory which has been consistent with a potentially positive relationship between inequality and growth in advanced countries so far rests on the classical view: that is, higher inequality may enhance growth through physical

\footnotetext{
${ }^{33}$ Usually, $Y_{0}$ has significant effects, suggesting that observed economies are not yet close to stationary equilibria.

${ }^{34}$ Banerjee and Duflo (2003) suggest that the change in inequality, in either direction, rather then its level is negatively associated with growth. Moreover, on basis of this finding, they argue that previous panel studies (particularly those relying on fixed effects) may have produced upward biased estimates of the effect of inequality on growth.

${ }^{35}$ For developing countries, a negative relationship between inequality and growth is consistent with the view that high inequality leads to social unrest (e.g., Alesina and Perotti, 1996), high fertility (e.g., Perotti, 1996), and low human capital investment (e.g., due to borrowing constraints; see Galor and Zeira, 1993), in turn, being associated with slow growth. Evidence by Perotti (1996) largely supports these channels.
} 
capital accumulation. However, this mechanism crucially depends on the assumption of (at least partially) closed economies such that investment in physical capital is related to national savings. In contrast, the theory proposed in this paper rests on the role of intergenerational wealth transmission for human capital accumulation in a small open economy. Abstracting from borrowing constraints for human capital investments renders the model particularly relevant for advanced economies.

The remainder of this section provides empirical evidence for the forces suggested by the present analysis which are necessary to obtain a growth-promoting medium run effect of inequality.

\subsection{Saving Behavior and Intergenerational Transfers}

In the model, the amount of intergenerational transfers equals the amount of savings of adult individuals. In fact, empirically, savings seem to be strongly related to inter vivos gifts and bequests, thus lending support for a crucial assumption about preferences in the model. For instance, Kotlikoff and Summers (1981) emphasize the importance of intergenerational transfers for capital accumulation in the US, a hypothesis which has been frequently confirmed by later evidence. In particular, as argued by Menchik and David (1983) and Dynan et al. (2000, 2002), observed saving behavior in the US is empirically consistent with models hypothesizing a "joy of giving" motive for intergenerational transfers. ${ }^{36}$ De Nardi (2003) calibrates an overlapping-generations model with voluntary and accidental bequests, arguing that voluntary bequests play a crucial role for explaining observed wealth concentration patterns not only in the US but also in Sweden.

According to the preceding analysis, if the marginal propensity to save for adults,

\footnotetext{
${ }^{36}$ In contrast, the standard altruism (dynastic) model á la Barro (1974) seems to be inconsistent with the data. For instance, unlike predicted by the dynastic model, inheritances do not seem to compensate for earnings differences among siblings (e.g. Wilhelm, 1996). Moreover, as discussed in Carroll (2000), there does not seem to be an indication that the size of bequest is an increasing function of the ratio of parent's to child's lifetime income. Finally, whereas a one-dollar reduction in income of a recipient should raise inter vivos transfers from parents to child by one dollar according to the dynastic model, evidence by Altonji et al. (1997) suggests that transfers increase by just 13 cent on average, conditional on the event of a positive transfer having occurred.
} 
$b^{\prime}(\cdot)$, is increasing in income, then inequality may be positively related to growth in the medium run, despite diminishing marginal returns to human capital investments (Proposition 3). This is because under individual uncertainty of returns to human capital investments, (initial) wealth inequality affects human capital accumulation through intergenerational transfers.

Evidence on US saving and bequest behavior suggests that saving rates are strongly increasing in lifetime income. ${ }^{37}$ For instance, Dynan et al. (2000) provide estimates (for the five-year period between 1984 and 1989) which imply that a $\$ 10,000$ increase in (permanent) income is associated with an increase in the saving rate in a range from over 1 to roughly 5 percent, depending on the database and instruments used. ${ }^{38}$ Most importantly for the results of the theoretical model developed in the present paper, estimates from median regressions suggest that the increase in saving rates in response to an increase in income is approximately constant. ${ }^{39}$ This implies that the marginal propensity to save is increasing as function of income, thus, being consistent with $b^{\prime \prime}(\cdot)>0$. In a less recent study, Menchik and David (1983) directly focus on bequest behavior. Their evidence suggests that the marginal propensity to bequeath is increasing in lifetime earnings.

Remark 1. It is important to note the difference between $b^{\prime \prime}(\cdot)>0$ on the one hand and an increasing average propensity of adults to save (which holds if $b(I) / I$ is increasing in $I$ ) on the other hand. For instance, following Galor and Zeira (1993), Moav (2002) and Galor and Moav (2004), among others, consider the saving function

\footnotetext{
${ }^{37}$ There is overwhelming evidence for the hypothesis that saving rates rise strongly with current income (e.g., Browning and Lusardi, 1996). However, as pointed out by Friedman (1957), this finding may just reflect a response of savings behavior to changes in transitory income. That is, if income is temporarily high, savings increase and, analogously, if income is temporarily low, savings are reduced. Over the life-cycle, however, a positive relationship between current income and saving rates may still be consistent with a constant saving rate as function of lifetime (or permanent) income.

${ }^{38}$ Dynan et al. (2000) use three different databases to account for different measures of savings and use different instruments for permanent income like consumption, lagged and/or future earnings, and education. Using current income, a $\$ 10,000$ increase in income is associated with a 8 percent increase in the saving rate. This figure is considerably higher than for measures of permanent income, as expected.

${ }^{39}$ See Dynan et al. (2000, Fig. 1A-1D). This result is basically independent on the instrument used or when no instrumenting is done.
} 
$b(I)=\alpha[I-\vartheta]$ if $I>\vartheta$ and $b(I)=0$ otherwise, $\vartheta \geq 0$. Thus, if $I \geq \vartheta>0$, the average propensity of adults to save, $b(I) / I=\alpha-\vartheta / I$, is increasing in $I$. Nevertheless, since $b^{\prime \prime}(I) \leq 0$ for $I>\vartheta$, this particular functional form rules out the potentially positive effect of inequality on medium run growth, suggested by part (ii) of Proposition 3. Appendix $\mathrm{C}$ further employs this saving function for an illustration of the analysis under a particular specification of preferences.

\subsection{Educational Investment and Parental Income}

Besides $b^{\prime \prime}(\cdot)>0$, a second necessary condition for a positive relationship between inequality and medium run growth in the model is the positive relationship between family wealth and educational investments, $e^{\prime}(\cdot)>0$ (Proposition 1$)$. This condition has been derived from the hypothesis that the variance of earnings increases with educational levels. In fact, there is overwhelming evidence for both this basic assumption and its implication.

First, evidence by Pereira and Martins $(2002,2003)$ for advanced European countries (in a period between 1980 and 1995) strongly suggests that the earnings dispersion is increasing in the level of schooling, confirming somewhat less recent evidence discussed in Levhari and Weiss (1974). Moreover, although credit constraints to finance higher education seem to be negligible in advanced countries, there is a strong positive relationship between parental social background and children's investment (or participation) in higher education. For instance, Manski (1992) finds that the percentage of children from low-income families in the US who graduate from high school is substantially lower than among high school graduates from other families. Similar patterns can be found in Germany. Egeln et al. (2003) report that 1996 even among those children who were eligible for university education (not more than roughly a third of all high school graduates in Germany), only 24 percent with a less favorable social background went to university, in contrast to 86 percent with a favorable social background. ${ }^{40}$ This

\footnotetext{
${ }^{40}$ In Germany, eligibility to attend university is exclusivley determined by high school performance. Egeln et al. (2003) also find that among all high school graduates only 8 percent with a less favorable social background went to university, in contrast to 72 percent of high school graduates with a favorable
} 
comparison is striking, as it is rather implausible that heterogeneity in intellectual ability (which may partly be shaped by the social background) can account for this difference among those who have already acquired eligibility ("Hochschulreife").

In his review of US evidence based on econometric studies, Taubman (1989) concludes that estimates for the elasticity of years of schooling with respect to parental income are generally positive and range from 3 to 80 percent, after controlling for parents' education, father's occupation, and/or children's test scores on mental ability tests. Accounting for similar controls, also the correlation between children's adult earnings and their parents' income is highly positive (e.g. Behrman and Taubman, 1990). As concluded in the survey article by Solon (1999, p.1789): "Most of the evidence $[. .$.$] indicates that intergenerational earnings elasticities are substantial and are$ larger than we used to think." The more recent literature on the relationship between a child's social environment and its educational attainment or earnings, respectively, is particularly concerned with the question to which degree such results are driven by genetic factors. For instance, Sacerdote (2002) finds that the effect of socioeconomic status on children's college attendance is just as large for adoptees as for children raised by biological parents. Plug and Vijverberg (2003) report higher effects of genetic factors (measured by parents' IQ) on the children's years of schooling and college attainment, although family income still has a large effect.

\section{Concluding Remarks}

After more than a decade of intensive research on the relationship between inequality and growth the debate is still ongoing. This paper has proposed a theory which is consistent with a positive medium run effect of inequality on growth in advanced countries, as found in Barro (2000). In contrast to the classical view, this alternative theory does not require any connection between national savings and physical capital investment in an economy, but rests on the role of intergenerational wealth transmission for social background. 
individual incentives to invest in risky and uninsurable human capital.

First, it has been shown that, under the fairly weak requirement of decreasing absolute risk aversion (defined over indirect utility), individual human capital investment is increasing in parental income. This prediction is largely supported by empirical evidence even in advanced countries (in which credit-market imperfections may play a minor role). Given this positive relationship between parental income and human capital investments, if the marginal return to education is diminishing, then the expected marginal return to education is higher for poorer individuals. Thus, in the short run, initial inequality of family wealth is typically negatively linked to the aggregate human capital stock, and thus, is an impediment for the short run process of development.

However, in the medium run, the effects of intergenerational wealth transmission may overturn this short run effect of inequality on growth if the marginal propensity to save is increasing in income. In fact, this property has frequently been confirmed. In contrast, if the marginal propensity to save is non-increasing, the model predicts that the relationship between inequality and medium run growth is typically negative.

For the long run (i.e., in stationary equilibrium), irrespective of the properties of the saving function, the relationship between inequality and per capita income (or the long run rate of growth, respectively, allowing for endogenous growth in a straightforward way) can go either way.

It is important to note, however, that even if the inequality-growth relationship turns out to be positive, the proposed theory does not suggest a rationale for inegalitarian policies. For instance, under uninsurable human capital risk, distortionary redistribution through the tax system may enhance risk-taking by providing insurance, as suggested by the literature on portfolio choice and taxation. ${ }^{41}$ To examine the role of the shape of the saving function for implications of redistributive taxation in a similar context as analyzed in this paper is left for future research.

\footnotetext{
${ }^{41}$ Moreover, since the model does not contain any "trickle-down" mechanism, a standard equitygrowth trade-off arises such that optimal policy will crucially hinge on the social welfare function.
} 


\section{Appendix}

\section{A. Proof of Proposition 1}

First, note that $e_{t}^{i}=e\left(b_{t}^{i}\right)$ is given by the first-order condition

$$
\begin{aligned}
0 & =E\left[v^{\prime}\left(I\left(b_{t}^{i}, e_{t}^{i}, \tilde{a}\right)\right)\left(\bar{w} h_{e}\left(e_{t}^{i}, \tilde{a}\right)-\bar{R}\right)\right], \text { i.e., } \\
\Xi\left(b_{t}^{i}, e_{t}^{i}\right) & \equiv E\left[v^{\prime}\left(\bar{w} h\left(e_{t}^{i}, \tilde{a}\right)+\bar{R}\left(b_{t}^{i}-e_{t}^{i}\right)\right)\left(\bar{w} h_{e}\left(e_{t}^{i}, \tilde{a}\right)-\bar{R}\right)\right]=0,
\end{aligned}
$$

according to (3) and (9). (An interior solution is ensured by assumption A1.) Due to $v^{\prime \prime}(I)<0$ (recall Lemma 2) and $h_{e e}<0$, we have $\Xi_{e}<0$. Thus, according to the implicit function theorem, $e^{\prime}\left(b_{t}^{i}\right)>0$ if and only if $\left.\Xi_{b}\left(b_{t}^{i}, e_{t}^{i}\right)\right|_{e_{t}^{i}=e\left(b_{t}^{i}\right)}>0$. For notational simplicity, indices $t$ and $i$ are suppressed in the remainder of this proof. Moreover, define $\hat{I}(b, \tilde{a}) \equiv I(b, e(b), \tilde{a})$. Then, according to (A.1),

$$
\begin{aligned}
\left.\Xi_{b}(b, e)\right|_{e=e(b)} & =E\left[v^{\prime \prime}(\hat{I}(b, \tilde{a}))\left(\bar{w} h_{e}(e(b), \tilde{a})-\bar{R}\right)\right] \bar{R} \\
& =E\left[A(\hat{I}(b, \tilde{a})) v^{\prime}(\hat{I}(b, \tilde{a}))\left(\bar{R}-\bar{w} h_{e}(e(b), \tilde{a})\right)\right] \bar{R}
\end{aligned}
$$

where $A(I)=-v^{\prime \prime}(I) / v^{\prime}(I)$ has been used for the latter equation. Define $a^{\prime}$ as the realization of $\tilde{a}$ such that $\bar{w} h_{e}\left(e, a^{\prime}\right)-\bar{R}=0$. We can write

$$
\begin{aligned}
& E\left[A(\hat{I}(b, \tilde{a})) v^{\prime}(\hat{I}(b, \tilde{a}))\left(\bar{R}-\bar{w} h_{e}(\cdot, \tilde{a})\right)\right] \\
= & \int_{\underline{a}}^{a^{\prime}} A(\hat{I}(b, \tilde{a})) v^{\prime}(\hat{I}(b, \tilde{a}))\left(\bar{R}-\bar{w} h_{e}(\cdot, \tilde{a})\right) d \Phi(\tilde{a})+ \\
& \int_{a^{\prime}}^{\bar{a}} A(\hat{I}(b, \tilde{a})) v^{\prime}(\hat{I}(b, \tilde{a}))\left(\bar{R}-\bar{w} h_{e}(\cdot, \tilde{a})\right) d \Phi(\tilde{a}) .
\end{aligned}
$$

Recall from assumption A1 that $h_{e a}>0$. Thus, by the definition of $a^{\prime}$, the first integral in (A.3) is positive, whereas the second one is negative. Moreover, note that since $h_{a}>0, \hat{I}(b, a)$ is increasing in $a$, according to $(3)$. Thus, $A(\hat{I}(b, a))$ is strictly 
decreasing in $a$ under assumption A3. Hence, under A3,

$$
\begin{aligned}
& A\left(\hat{I}\left(b, a^{\prime}\right)\right) \int_{\underline{a}}^{a^{\prime}} v^{\prime}(\hat{I}(b, \tilde{a}))\left(\bar{R}-\bar{w} h_{e}(\cdot, \tilde{a})\right) d \Phi(\tilde{a}) \\
< & \int_{\underline{a}}^{a^{\prime}} A(\hat{I}(b, \tilde{a})) v^{\prime}(\hat{I}(b, \tilde{a}))\left(\bar{R}-\bar{w} h_{e}(\cdot, \tilde{a})\right) d \Phi(\tilde{a}), \\
& A\left(\hat{I}\left(b, a^{\prime}\right)\right) \int_{a^{\prime}}^{\bar{a}} v^{\prime}(\hat{I}(b, \tilde{a}))\left(\bar{R}-\bar{w} h_{e}(\cdot, \tilde{a})\right) d \Phi(\tilde{a}) \\
< & \int_{a^{\prime}}^{\bar{a}} A(\hat{I}(b, \tilde{a})) v^{\prime}(\hat{I}(b, \tilde{a}))\left(\bar{R}-\bar{w} h_{e}(\cdot, \tilde{a})\right) d \Phi(\tilde{a}) .
\end{aligned}
$$

Adding up (A.4) and (A.5) and using $A(I) v^{\prime}(I)=-v^{\prime \prime}(I)$ yields

$$
A\left(\hat{I}\left(b, a^{\prime}\right)\right) E\left[v^{\prime}(\hat{I}(b, \tilde{a}))\left(\bar{R}-\bar{w} h_{e}(\cdot, \tilde{a})\right)\right]<E\left[v^{\prime \prime}(\hat{I}(b, \tilde{a}))\left(\bar{w} h_{e}(\cdot, \tilde{a})-\bar{R}\right)\right]
$$

Under the optimal human capital investment, $e(b)$, the left-hand side of (A.6) is zero, according to (A.1). Thus, $E\left[v^{\prime \prime}(\hat{I}(b, \tilde{a}))\left(\bar{w} h_{e}(\cdot, \tilde{a})-\bar{R}\right)\right]>0$, implying $\left.\Xi_{b}(b, e)\right|_{e=e(b)}>$ 0 , according to (A.2). Hence, $e^{\prime}(b)>0$. This concludes the proof.

\section{B. Stationary Equilibria}

This appendix provides a more formal treatment of stationary equilibria and generalizes the cases discussed in section 4.2 (based on Fig. 1).

Let $P(b, \cdot)$ be the transition function of the Markov process $b_{t+1}^{i}=\hat{b}\left(b_{t}^{i}, \tilde{a}\right)$, i.e., $P\left(b^{i}, \mathcal{Z}\right)$ is the probability that $b^{i}$ is in the set $\mathcal{Z}$ one period after it started in $b^{i}$. That is,

$$
P\left(b^{i}, \mathcal{Z}\right) \equiv \operatorname{Pr}\left\{\tilde{a}: \hat{b}\left(b^{i}, \tilde{a}\right) \in \mathcal{Z}\right\} \equiv \operatorname{Pr}\left\{\tilde{a} \in \mathcal{Z}_{b^{i}}\right\}=\int_{\mathcal{Z}_{b^{i}}} \Phi(\tilde{a}),
$$

where $\mathcal{Z}_{b^{i}} \equiv\left\{\tilde{a}: \hat{b}\left(b^{i}, \tilde{a}\right) \in \mathcal{Z}\right\}$ and $\mathcal{Z}$ is a Borel set in $\mathbb{R}_{+}$. Moreover, let $\mu_{t}^{i}(\mathcal{Z}) \equiv$ $\operatorname{Pr}\left\{b_{t}^{i} \in \mathcal{Z}\right\}$ for all $\mathcal{Z} \subset \mathbb{R}_{+}, t=0,1,2, \ldots$, be the probability measure associated with $b_{t}^{i}$. Thus, given initial wealth $b_{0}^{i}$ of dynasty $i, \mu_{0}^{i}$ is given by $\mu_{0}^{i}\left(\left[0, b^{i}\right)\right)=0$ for all 
$b^{i} \leq b_{0}^{i}$ and $\mu_{0}^{i}\left(\left[b^{i}, \infty\right)\right)=1$ otherwise. Starting from $\mu_{0}^{i}$, the distribution of family wealth evolves according to $\mu_{t+1}^{i}(\mathcal{Z})=\int P\left(b^{i}, \mathcal{Z}\right) \mu_{t}^{i}\left(d b^{i}\right)$ for all $\mathcal{Z} \subset \mathbb{R}_{+}$. From this, we can define a stationary equilibrium as follows. ${ }^{42}$

Definition 1. (Stationary equilibrium). A stationary equilibrium for family wealth $b^{i}$ of dynasty $i$ is a probability measure $\mu^{i}$ such that $\mu^{i}(\mathcal{Z})=\int P\left(b^{i}, \mathcal{Z}\right) \mu^{i}\left(d b^{i}\right)$ for all $\mathcal{Z} \subset \mathbb{R}_{+}$. A trivial stationary equilibrium is a stationary equilibrium which is associated with a distribution of $b^{i}$ such that all mass is concentrated on zero (i.e., $\left.\lim _{t \rightarrow \infty} \operatorname{Pr}\left\{b_{t}^{i}=0\right\}=1\right)$.

Together with Definition 1, the next definition leads to an important existence result.

Definition 2. (Stable set). An interval $[x, y] \subset \mathbb{R}$ is called a stable set of the stochastic process $\hat{b}$ if (i) $\hat{b}\left(b^{i}, x\right)=x, \hat{b}\left(b^{i}, y\right)=y$, and (ii) $\hat{b}\left(b^{i}, x\right)<b^{i}, \hat{b}\left(b^{i}, y\right)>b^{i}$ for all $x<b^{i}<y$.

Lemma B.1. (Wang, 1993). There is a unique stable stationary equilibrium on a stable set I. Moreover, the convergence to the stationary equilibrium is uniform on $I$.

Proof. Brock and Mirman (1972), Wang (1993).

Given these preliminaries, let $S_{a} \equiv\left\{b \in \mathbb{R}_{++} \mid \hat{b}(b, a)=0\right\}$ be the set of strictly positive transfers received by a young individual, such that the optimal transfer as adult to her offspring is zero, $a \in \mathcal{A}$. Moreover, let $\underline{b}_{a} \equiv \max S_{a}$ be the largest element of such a set. Suppose that the following holds.

A6. $S_{\bar{a}}$ is non-empty, i.e., there exists $\underline{b}_{\bar{a}}>0$.

Note that $\hat{b}_{a}(b, a)=b^{\prime}(\hat{I}(b, a)) \hat{I}_{a}$, according to (12) and (13). Thus, using $\hat{I}_{a}>0$ and Lemma 1 , we have $\underline{b}_{a}>\underline{b}_{\bar{a}}$ for all $a \in[\underline{a}, \bar{a})$. That is, if an individual which has received a transfer $b$ when young does not save as adult in the best state $\bar{a}$, neither she

\footnotetext{
${ }^{42}$ Definitions 1 and 2 closely follow Wang (1993).
} 
does in any other state. Also note that, if $\underline{b}_{a}>0$ exists for some $a$, then A2 and A5 imply that $\hat{b}(b, a)>0$ and $\hat{b}_{b}(b, a)>0$ for all $b>\underline{b}_{a}$, according to Lemma 3 .

We are now ready to give a formal characterization of the result corresponding to panel (a) of Fig. 1.

Proposition B.1. Under A2, A5 and A6. If $\hat{b}\left(b^{i}, \bar{a}\right)<b^{i}$ for all $b^{i}>0$, the distribution of $b^{i}$ globally converges to a trivial stationary equilibrium.

Proof. First, note that A6, which says that $S_{\bar{a}}$ is non-empty, also implies that $S_{\underline{a}}$ is non-empty. Moreover, recall $\underline{b}_{a}>\underline{b}_{\bar{a}}>0$. Also recall that A2, A5 and A6 ensure $\hat{b}(b, a)>0$ and $\hat{b}_{b}(b, a)>0$ for all $b>\underline{b}_{a}$, according to Lemma 3. Thus, Proposition B.1 corresponds to the case in panel (a) of Fig. 1. From this figure, it is clear that there no stable set of the process $\hat{b}$ exists (recall Definition 2), and global convergence to a trivial stationary equilibrium (recall Definition 1 ) is obvious.

To analyze more general situations than global convergence to a trivial stationary equilibrium, the next assumption prevents infinite wealth accumulation of rich dynasties.

A7. $\lim _{b \rightarrow \infty} \hat{b}_{b}(b, a)=0$ for all $a \in \mathcal{A}$.

Moreover, let $\Theta_{a} \equiv\left\{b \in \mathbb{R}_{++} \mid \hat{b}(b, a)=b\right\}$ be the set of strictly positive fixed points of $\hat{b}(b, a), a \in \mathcal{A}$. In the remainder of this appendix, we focus on situations in which $\Theta_{\underline{a}}$ and $\Theta_{\bar{a}}$ have the following properties.

A8. (i) $\Theta_{\underline{a}}$ is non-empty. ${ }^{43}$ (ii) $\Theta_{\underline{a}}$ and $\Theta_{\bar{a}}$ are finite. (iii) Let $c_{\bar{a}}, d_{\bar{a}}$ be two adjacent elements of $\Theta_{\bar{a}}$ such that $c_{\bar{a}}<d_{\bar{a}}$ and $\hat{b}_{b}\left(d_{\bar{a}}, \bar{a}\right) \leq 1$. Then there exists $c_{\underline{a}} \in \Theta_{\underline{a}}$ such that $c_{\underline{a}} \in\left(c_{\bar{a}}, d_{\bar{a}}\right)$. (iv) Let $c_{\underline{a}}, d_{\underline{a}}$ be two adjacent elements of $\Theta_{\underline{a}}$ such that $c_{\underline{a}}<d_{\underline{a}}$ and $\hat{b}_{b}\left(d_{\underline{a}}, \underline{a}\right) \geq 1$. Then there exists $c_{\bar{a}} \in \Theta_{\bar{a}}$ such that $c_{\bar{a}} \in\left(c_{\underline{a}}, d_{\underline{a}}\right)$.

\section{$<$ Figure 2 $>,<$ Figure $3>$}

\footnotetext{
${ }^{43}$ Note that, under A6, part (i) of A8 implies that $\Theta_{\bar{a}}$ is non-empty as well.
} 
It is easy to check that the case depicted in panel (b) of Fig. 1 is consistent with A6A8. ${ }^{44}$ Fig. 2 shows situations, which are consistent with A6 and A7, but inconsistent with some parts of A8, whereas panels (a)-(c) of Fig. 3 are, like panel (b) of Fig. 1, consistent with A6-A8.

Note that, by applying Definition 2, $I_{3}$ and $I_{6}$ in panels (a) and (b) of Fig. 3 are both stable sets, whereas in panel (c) $I_{3}$ is the unique stable set. Moreover, by replicating the arguments in Laitner (1981; section III), the following can be concluded from Fig. 3, starting with panels (a) and (b). In panel (a) of Fig. 3, if $b_{0}^{i} \in I_{2} \cup I_{3} \cup I_{4}$, then $\lim _{t \rightarrow \infty} \operatorname{Pr}\left\{b_{t}^{i} \in I_{3}\right\}=1$. In panel (b), the same is true if $b_{0}^{i} \in I_{2} \cup I_{3}$. In panel (a), if $b_{0}^{i} \in I_{6} \cup I_{7}$, then $\lim _{t \rightarrow \infty} \operatorname{Pr}\left\{b_{t}^{i} \in I_{6}\right\}=1$. In panel (b), the same is true if $b_{0}^{i} \in I_{5} \cup I_{6} \cup I_{7}$. In both panels (a) and (b), if $b_{0}^{i} \in I_{0}$, then $q \equiv \lim _{t \rightarrow \infty} \operatorname{Pr}\left\{b_{t}^{i}=0\right\}=1$, and, if $b_{0}^{i} \in I_{1}$, then $q \in(0,1)$ and $\lim _{t \rightarrow \infty} \operatorname{Pr}\left\{b_{t}^{i} \in I_{3}\right\}=1-q$. Finally, if $b_{0}^{i} \in I_{5}$ in panel (a), then $b_{t}^{i} \in I_{3}$ or $b_{t}^{i} \in I_{6}$ with probability one. In panel (b), the same is true if $b_{0}^{i} \in I_{4}$. Now consider panel (c) of Fig. 3. If $b_{0}^{i} \in I_{0} \cup I_{1}$, then $q=1$. If $b_{0}^{i} \in I_{2}$, then $q \in(0,1)$ and $\lim _{t \rightarrow \infty} \operatorname{Pr}\left\{b_{t}^{i} \in I_{3}\right\}=1-q$. Finally, if $b_{0}^{i} \in I_{3} \cup I_{4}$, then $\lim _{t \rightarrow \infty} \operatorname{Pr}\left\{b_{t}^{i} \in I_{3}\right\}=1$. Using this discussion, one can generalize the conclusions drawn from panel (b) of Fig. 1 discussed in the main text, in the following sense.

Proposition B.2. Under A2, A5-A8. Let $c=\min \Theta_{\bar{a}}$ and $d=\min \Theta_{\underline{a}}$ (i.e., $c<d)$.

(i) For all $b_{0}^{i} \in[0, c]$, as $t \rightarrow \infty$, the distribution of $b_{t}^{i}$ converges to a locally stable trivial stationary equilibrium.

(ii) For all $b_{0}^{i} \in(c, d)$, there is a positive probability $q \in(0,1)$ that the distribution of $b_{t}^{i}$ converges to a trivial stationary equilibrium, whereas with probability $1-q$ it converges to a (unique and stable) stationary equilibrium on a stable set.

(iii) For all $b_{0}^{i} \in[d, \infty)$, the distribution of $b^{i}$ converges to a (unique and stable) stationary equilibrium on a stable set.

Proof. Part (i) of Proposition B.2 can be deduced by similar arguments as in the proof of Proposition B.1. To prove parts (ii) and (iii), recall Definitions 1 and 2 and

\footnotetext{
${ }^{44}$ Note that part (iv) of A8 is not relevant for panel (b) of Fig. 1 , since $\hat{b}_{b}\left(d_{\underline{a}}, \underline{a}\right)<1$.
} 
verify from A2 and A5 (which ensure $\hat{b}(b, a)>0$ and $\hat{b}_{b}(b, a)>0$ for all $b>\underline{b}_{a}$ ) as well as A6-A8, that the result can directly be deduced by replicating the discussion of Fig. 3 above.

\section{A Simple Example}

This appendix provides a simple illustration of the analysis by specifying preferences. Following Galor and Zeira (1993), Moav (2002) and Galor and Moav (2004), among others, the utility is given by

$$
u\left(c_{t+1}^{i}, b_{t+1}^{i}\right)=(1-\alpha) \ln c_{t+1}^{i}+\alpha \ln \left(\gamma+b_{t+1}^{i}\right), \quad 0<\alpha<1, \gamma \geq 0
$$

It is easy to check that assumption A2 and thus Lemma 1 and 2 holds. Using the firstorder condition (6) for optimal savings as adult, (A.8) implies a saving function $b_{t+1}^{i}=$ $b\left(I_{t+1}^{i}\right)=\alpha\left[I_{t+1}^{i}-\vartheta\right]$ if $I_{t+1}^{i}>\vartheta \equiv \gamma(1-\alpha) / \alpha$ and $b_{t+1}^{i}=0$ otherwise. (See also Remark 1 in section 5.2.) Moreover, indirect utility is given by $v\left(I_{t+1}^{i}\right)=2 \ln \left(I_{t+1}^{i}+\gamma\right)+\eta$, where $\eta \equiv \alpha \ln \alpha+(1-\alpha) \ln (1-\alpha)$. Thus, the degree of absolute risk aversion is given by $A(I)=-v^{\prime \prime}(I) / v^{\prime}(I)=1 /(I+\gamma)$. That is, $A(I)$ is strictly decreasing in $I$, in line with assumption A3. Hence, $e^{\prime}(b)>0$, according to Proposition 1, where $e(b)$ is given by

$$
\int_{\mathcal{A}} \frac{\bar{w} h_{e}(e, \tilde{a})-\bar{R}}{\bar{w} h(e, \tilde{a})+\bar{R}(b-e)} d \Phi(\tilde{a})=0
$$

according to the first-order condition (A.1) for optimal educational investment. Using (A.9), tedious derivations reveal that the sign of $e^{\prime \prime}(b)$ is ambiguous, lending some justification to $\left|e^{\prime \prime}(b)\right| \approx 0$ (assumption A4). Regarding the short run, this means that $\hat{H}_{1}^{\prime}(\varepsilon)>0$ (Proposition 2) is likely to hold. For the remainder of this appendix, suppose $\gamma>0$, i.e., $\vartheta>0$. For the medium run and long run analysis, using (12), one then obtains

$$
b_{t+1}^{i}=\hat{b}\left(b_{t}^{i}, a\right)=\tilde{b}_{1}\left(b_{t}^{i}\right)=\left\{\begin{array}{c}
\alpha\left[\bar{w} h\left(e\left(b_{t}^{i}\right), a\right)+\bar{R}\left(b_{t}^{i}-e\left(b_{t}^{i}\right)\right)-\vartheta\right] \text { if } b_{t}^{i} \geq \underline{b}_{a} \\
0 \text { otherwise }
\end{array}\right.
$$


where $\underline{b}_{a}$ is given by $\bar{w} h\left(e\left(\underline{b}_{a}\right), a\right)+\bar{R}\left(\underline{b}_{a}-e\left(\underline{b}_{a}\right)\right)=\vartheta, a \in \mathcal{A}$. Note that A5 implies that $\underline{b}_{a}$ is unique, and A6 implies that $\underline{b}_{a}>0$. Thus, if $\alpha[\bar{w} h(e(b), a)+\bar{R}(b-e(b))-\vartheta]<b$ for all $a \in \mathcal{A}$ and for all $b>0$, Proposition A.1 applies, i.e., the distribution of $b^{i}$ globally converges to a trivial stationary equilibrium. Moreover, it is easy to check that, under assumption A4, (A.10) implies

$$
\hat{b}_{b b}\left(b_{t}^{i}, a\right)=\tilde{b}_{1}^{\prime \prime}(b)<0 \text { for all } b>\underline{b}_{a}, a \in \mathcal{A}
$$

Since $b^{\prime \prime}(I)=0$ for $I>\vartheta$ under utility specification (A.8), $\tilde{b}_{1}^{\prime \prime}(b)<0$ illustrates part (i) of Lemma 4. Thus, the impact of higher equality on medium run growth is positive, according to part (i) of Proposition 3. For the long run analysis, note that (A.11) is consistent with assumption A7. Moreover, if there exists a $b>0$ such that $\alpha[\bar{w} h(e(b), \underline{a})+\bar{R}(b-e(b))-\vartheta]=b$, i.e., if part (i) of A8 holds, (A.11) implies that also parts (ii)-(iv) of A8 hold. This illustrates that, for the long run, the discussion of Fig. 1 in the main text applies (Proposition 4).

\section{References}

Aghion, Philippe and Patrick Bolton (1997). A Theory of Trickle-down Growth and Development, Review of Economic Studies 64, 151-172.

Aghion, Philippe, Eve Caroli and Cecilia García-Peñalosa (1999). Inequality and Economic Growth: The Perspective of New Growth Theories, Journal of Economic Literature 37, 1615-1660.

Alesina, Alberto and Dani Rodrik (1994). Distributive Politics and Economic Growth, Quarterly Journal of Economics 109, 465-490.

Alesina, Alberto and Roberto Perotti (1996). Income Distribution, Political Instability, and Investment, European Economic Review 40, 1203-1228.

Altonji, Joseph G., Fumio Hayashi, and Laurence J. Kotlikoff (1997). Parental Altruism and Inter Vivos Transfers: Theory and Evidence, Journal of Political Economy 
$105,1121-66$.

Arrow, Kenneth J. (1971). Essays in the Theory of Risk-Bearing, North-Holland, Amsterdam.

Banerjee, Abhijit V. and Esther Duflo (2003). Inequality and Growth: What Can the Data Say?, Journal of Economic Growth 8, 267-299.

Banerjee, Abhijit V. and Andrew F. Newman (1991). Risk-Bearing and the Theory of Income Distribution, Review of Economic Studies 58, 211-235.

Banerjee, Abhijit V. and Andrew F. Newman (1993). Occupational Choice and the Process of Development, Journal of Political Economy 101, 274-298.

Barro, Robert J. (1974). Are Government Bonds Net Wealth? Journal of Political Economy 82, 1095-1117.

Barro, Robert J. (1991). Economic Growth in a Cross-Section of Countries, Quarterly Journal of Economics 106, 407-444.

Barro, Robert J. (2000). Inequality and Growth in a Panel of Countries, Journal of Economic Growth 5, 5-32.

Becker, Gary and Nigel Tomes (1986). Human Capital and the Rise and Fall of Families, Journal of Labor Economics 4, S1-S39.

Behrman, Jere R. and Paul Taubman (1990). The Intergenerational Correlation between Children's Adult Earnings and their Parents' Income: Results from the Michigan Panel Survey of Income Dynamics, Review of Income and Wealth 36, 115-127.

Bénabou, Roland (1996). Inequality and Growth, NBER Macroeconomics Annual, MIT Press, Cambridge, MA, 11-74.

Bénabou, Roland (2002). Tax and Education Policy in a Heterogenous-Agent Economy: What Levels of Redistribution Maximize Growth and Welfare, Econometrica 70, 481-519.

Bourguignon, Francois (1981). Pareto Superiority of Unegalitarian Equilibria in Stiglitz' Model of Wealth Distribution With Convex Saving Function, Econometrica 49 (6), 1469-1475.

Brock, William A. and Leonard Mirman (1972). Optimal Economic Growth and 
Uncertainty: The Discounted Case, Journal of Economic Theory 4, 479-513.

Browning, Martin and Annamaria Lusardi (1996). Household Saving: Micro Theory and Micro Facts, Journal of Economic Literature 34, 1797-1855.

Carroll, Christopher D. (2000). Why Do the Rich Save So Much, in: J. B. Slemrod (ed.), Does Atlas Shrug? The Economic Consequences of Taxing the Rich, Harvard University Press, Cambridge, MA.

Carroll, Christopher D. (2002). Portfolios of the Rich, in: L. Guiso, M. Haliassos and T. Jappelli (eds.), Household Portfolios, MIT Press, Cambridge, MA.

Deininger, Klaus and Lyn Squire (1998). New Ways of Looking at Old Issues: Inequality and Growth, Journal of Development Economics 57, 259-287.

De Nardi, Mariacristina (2003). Wealth Inequality and Intergenerational Links, Review of Economic Studies (forthcoming).

Dynan, Karen E., Jonathan Skinner, and Stephen P. Zeldes (2000). Do the Rich Save More?, NBER Working Paper No. 7906.

Dynan, Karen E., Jonathan Skinner, and Stephen P. Zeldes (2002). The Importance of Bequests and Life-Cycle Saving in Capital Accumulation: A New Answer, American Economic Review Papers and Proceedings 92, 274-278.

Eaton and Rosen (1980). Taxation, Human Capital, and Uncertainty, American Economic Review 70, 705-715.

Egeln, Jürgen et al. (2003). Indikatoren zur Ausbildung im Hochschulbereich: Studie zum Innovationssystem Deutschlands, No. 10-2003 (Report to the German Ministry of Education and Research), Centre for European Economic Research (ZEW). Fishman, Arthur and Avi Simhon (2002). The Division of Labor, Inequality and Growth, Journal of Economic Growth 7, 117-136.

Forbes, Kristin (2000). A Reassessment of the Relationship Between Inequality and Growth, American Economic Review 90, 869-887.

Friedman, Milton (1957). A Theory of the Consumption Function, Princeton University Press, Princeton, NJ.

Galor, Oded and Joseph Zeira (1993). Income Distribution and Macroeconomics, 
Review of Economic Studies 60, 35-52.

Galor, Oded and Daniel Tsiddon (1997). The Distribution of Human Capital and Economic Growth, Journal of Economic Growth 2, 93-124.

Galor, Oded and Omer Moav (2004). From Physical to Human Capital Accumulation: Inequality in the Process of Development, Review of Economic Studies (forthcoming).

Glomm, Gerhard and B. Ravikumar (1992). Public versus Private Investment in Human Capital: Endogenous Growth and Income Inequality, Journal of Political Economy 100, 818-834.

Goldin Claudia and Lawrence F. Katz (1998). The Origins of Technology-Skill Complementarity, Quarterly Journal of Economics 113, 693-732

Gollier, Christian (2001). The Economics of Risk and Time, MIT Press, Cambridge, MA.

Gould, Eric D., Omer Moav and Bruce A. Weinberg (2001). Precautionary Demand for Education, Inequality, and Technological Progress, Journal of Economic Growth 6, $285-315$.

Grossmann, Volker (2001). Inequality, Economic Growth, and Technological Change: New Aspects in an Old Debate, Doctoral Dissertation, Physika, Heidelberg.

Krebs, Tom (2003). Human Capital Risk and Economic Growth, Quarterly Journal of Economics 118, 709-744.

Kotlikoff, Laurence J. and Lawrence H. Summers (1981). The Role of Intergenerational Transfers in Aggregate Capital Accumulation, Journal of Political Economy 89, 706-732.

Laitner, John (1981). The Steady States of a Stochastic Decentralized Growth Model, Journal of Economic Theory 24, 377-392.

Levhari, David and Yoram Weiss (1974). The Effect of Risk on Investment in Human Capital, American Economic Review 64, 950-963.

Li, Hongyi and Heng-fu Zou (1998). Income Inequality is Not Harmful for Growth, Review of Development Economics 2, 318-334. 
Manski, Charles (1992). Parental Income and College Opportunity, Democratic Study Center Report, Washington, D.C.

Menchik, Paul L. and Martin David (1983). Income Distribution, Lifetime Savings, and Bequests, American Economic Review 73, 672-690.

Moav, Omer (2002). Income Distribution and Macroeconomics: The Persistence of Inequality in a Convex Technology Framework, Economics Letters 75, 187-192.

Pereira, Pedro T. and Pedro S. Martins (2002). Is There a Return-Risk Link in Education? Economic Letters 75, 31-37.

Pereira, Pedro T. and Pedro S. Martins (2003). Does Education Reduce Wage Inequality? Quantile Regression Evidence from 16 Countries, Labour Economics (forthcoming).

Perotti, Roberto (1993). Political Equilibrium, Income Distribution, and Growth, Review of Economic Studies 60, 755-776.

Perotti, Roberto (1996). Growth, Inequality, and Democracy: What the Data Say, Journal of Economic Growth 1, 149-187.

Persson, Torsten and Guido Tabellini (1994). Is Inequality Harmful for Growth?, American Economic Review 84, 600-621.

Plug, Erik and Wim Vijverberg (2003). Schooling, Family Background, and Adoption: Is It Nature or Is It Nurture?, Journal of Political Economy 111 (3), 611-641.

Sacerdote, Bruce (2002). The Nature and Nuture of Economic Outcomes, American Economic Review Papers and Proceedings 92, 344-348.

Solon, Gary (1999). Intergenerational Mobility in the Labor Market, in: Ashenfelter, O. and D. Card, Handbook of Labor Economics, Vol. 3A, ch. 29, Elsevier, Amsterdam et al., 1761-1800.

Stiglitz, Joseph E. (1969). Distribution of Income and Wealth Among Individuals, Econometrica 37, 382-397.

Taubman, Paul (1989). Role of Parental Income in Educational Attainment, American Economic Review Papers and Proceedings 79, 57-61.

Venieris, Yiannis P. and Dipak K. Gupta (1986). Income Distribution and So- 
ciopolitical Instability as Determinants of Savings: A Cross-sectional Model, Journal of Political Economy 94, 873-883.

Wang, Yong (1993). Stationary Equilibria in an Overlapping Generations Economy with Stochastic Production, Journal of Economic Theory 61, 423-435.

Wildasin, David (2000). Labor-Market Integration, Investment in Risky Human Capital, and Fiscal Competition, American Economic Review 90, 73-95.

Wilhelm, Mark O. (1996). Bequest Behavior and the Effect of Heirs' Earnings: Testing the Altruistic Model of Bequests, American Economic Review 86, 874-892.

Zweimüller, Josef (2000). Schumpeterian Entrepreneurs Meet Engel's Law: The Impact of Inequality on Innovation-Driven Growth, Journal of Economic Growth 5, 185-206. 
Figure 1: Stationary equilibrium and convergence.

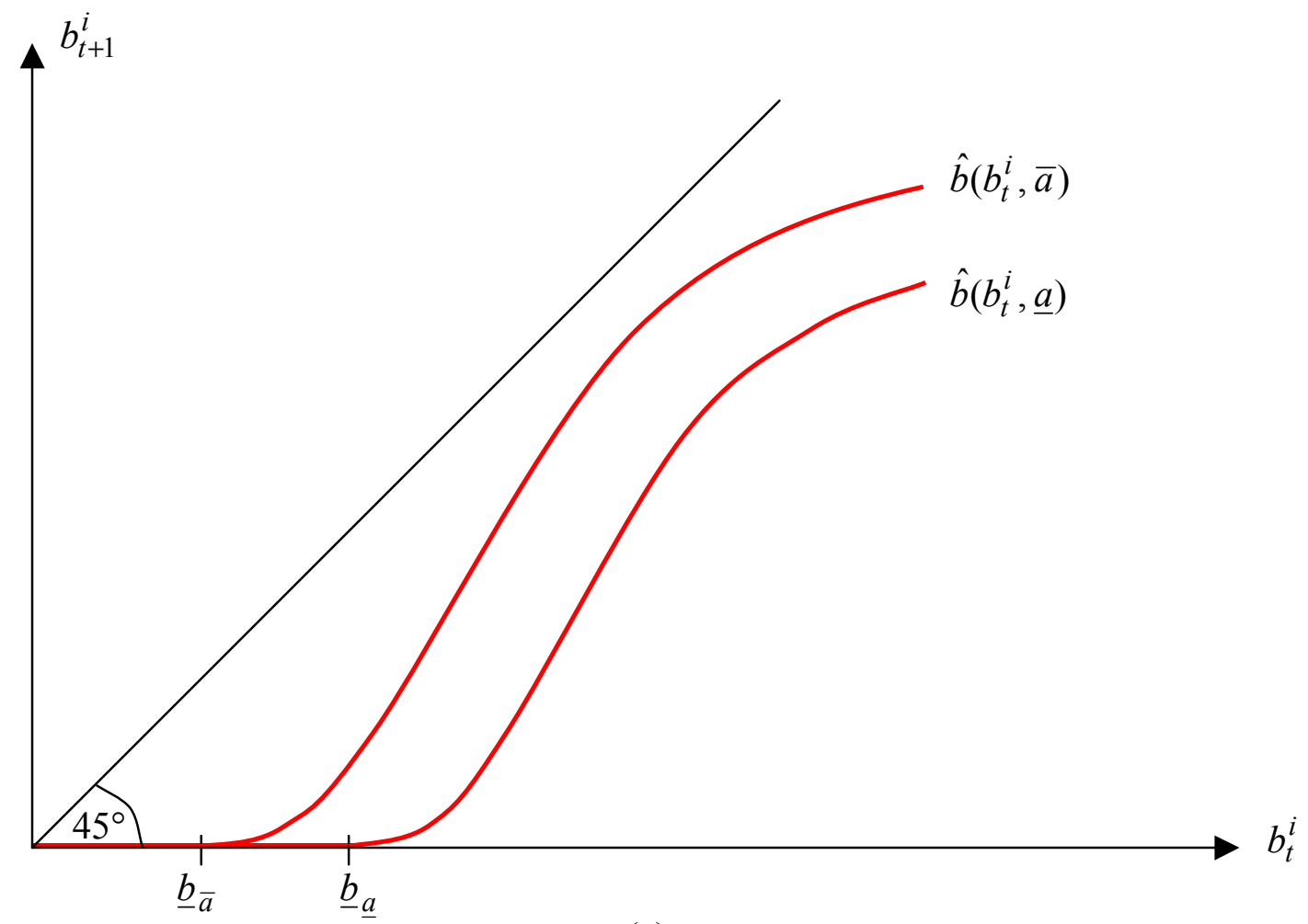

(a)

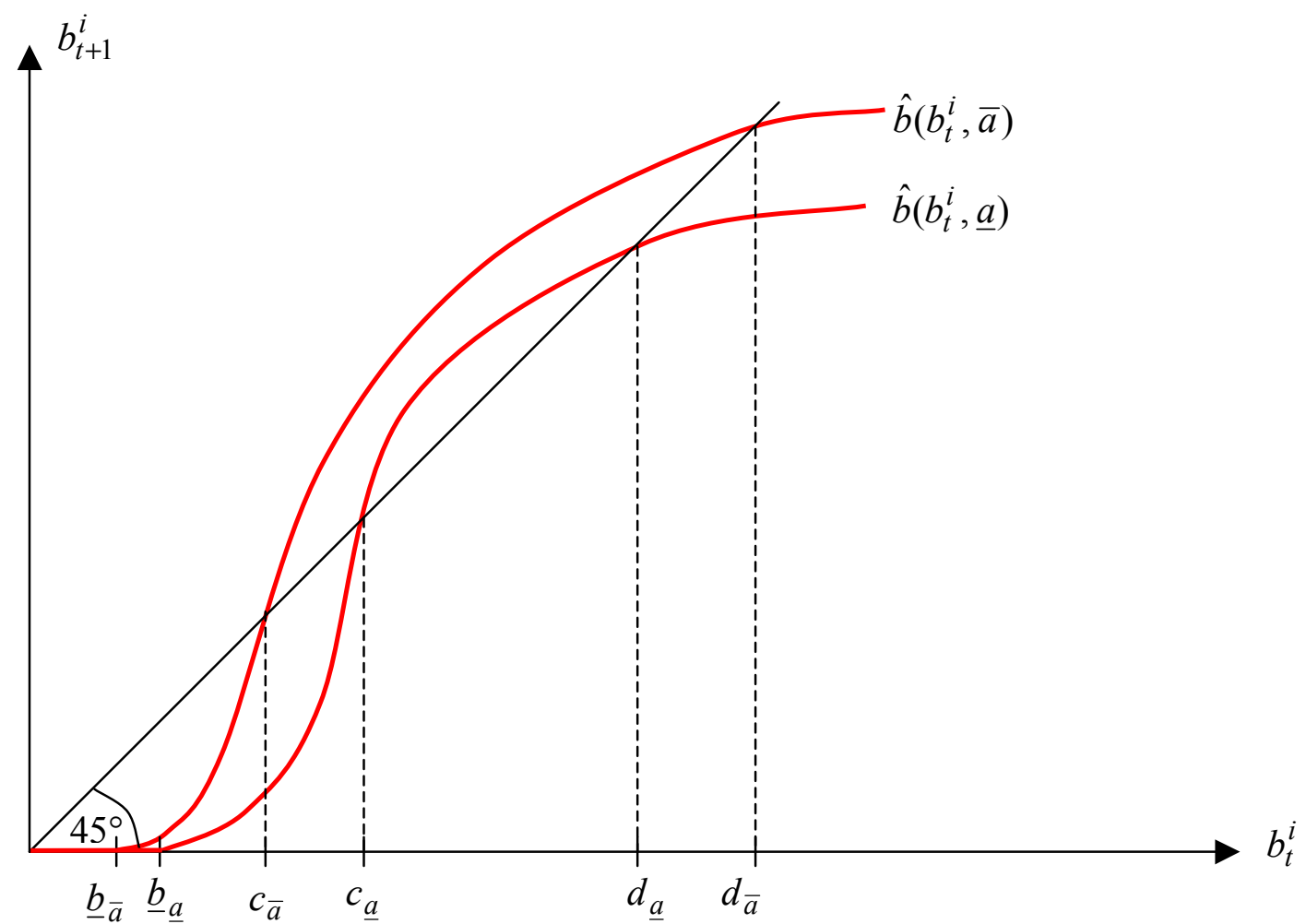

(b) 
Figure 2: Inconsistency with assumption A8.

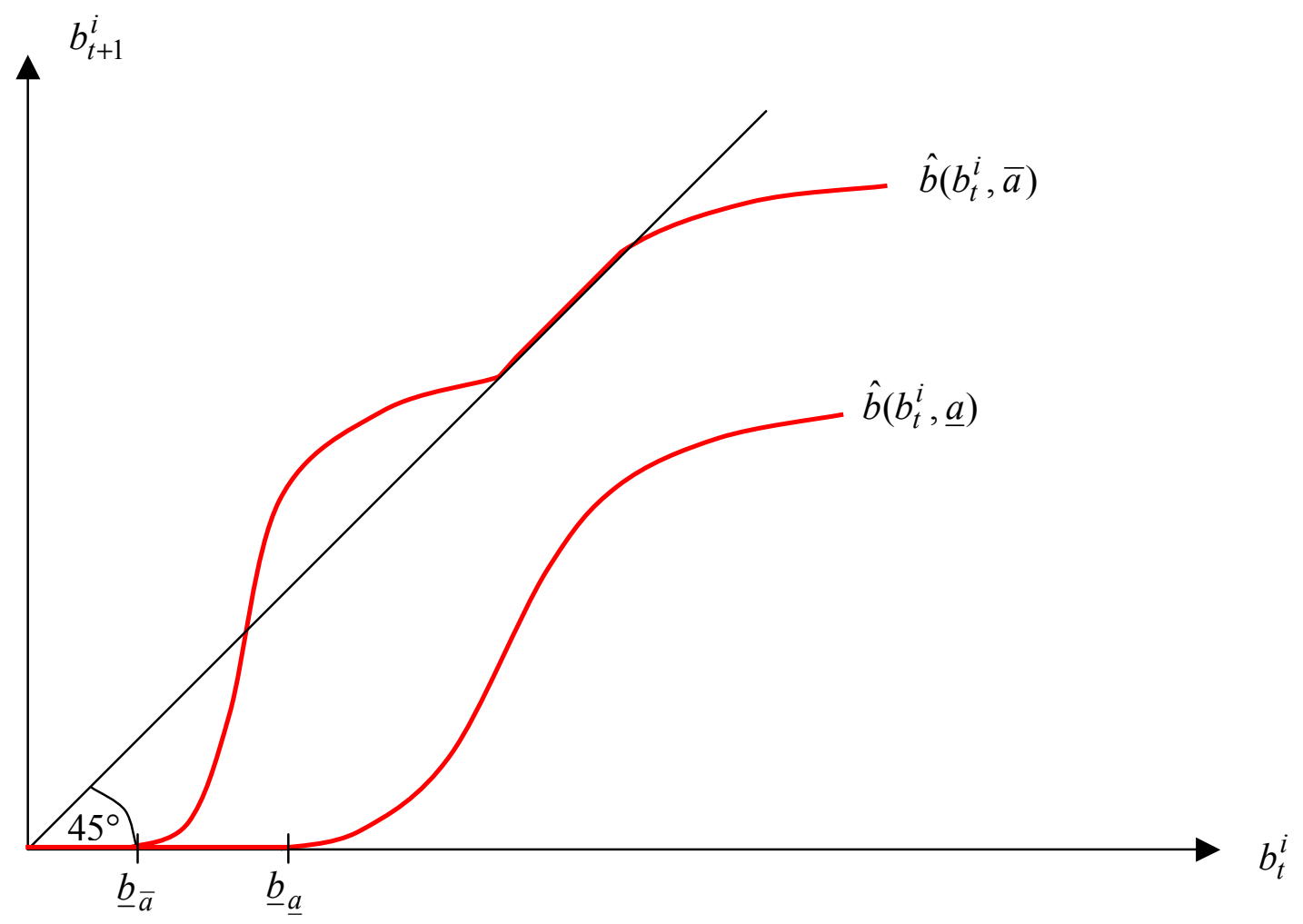

(a)

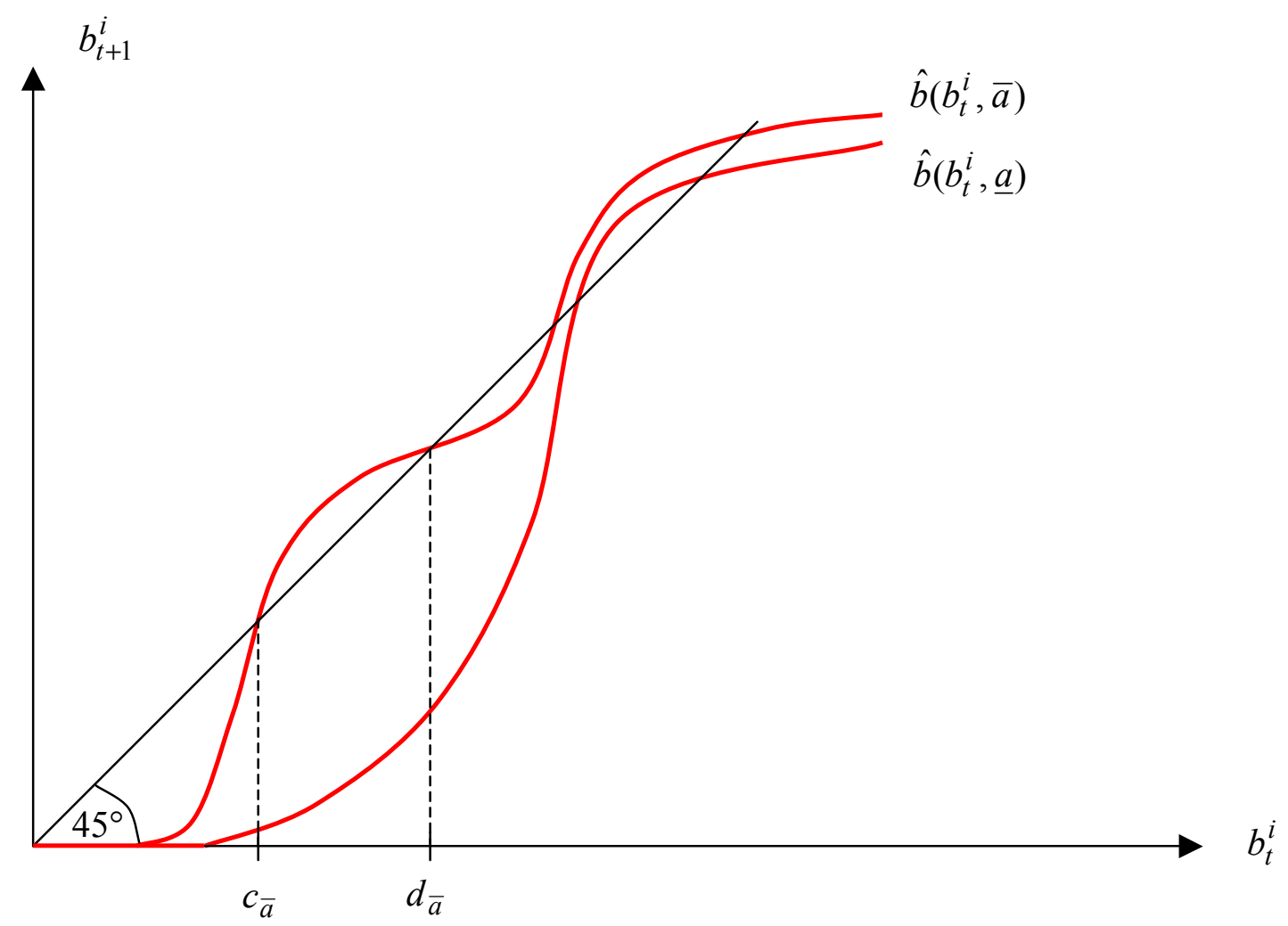

(b) 
(Figure 2 continued)

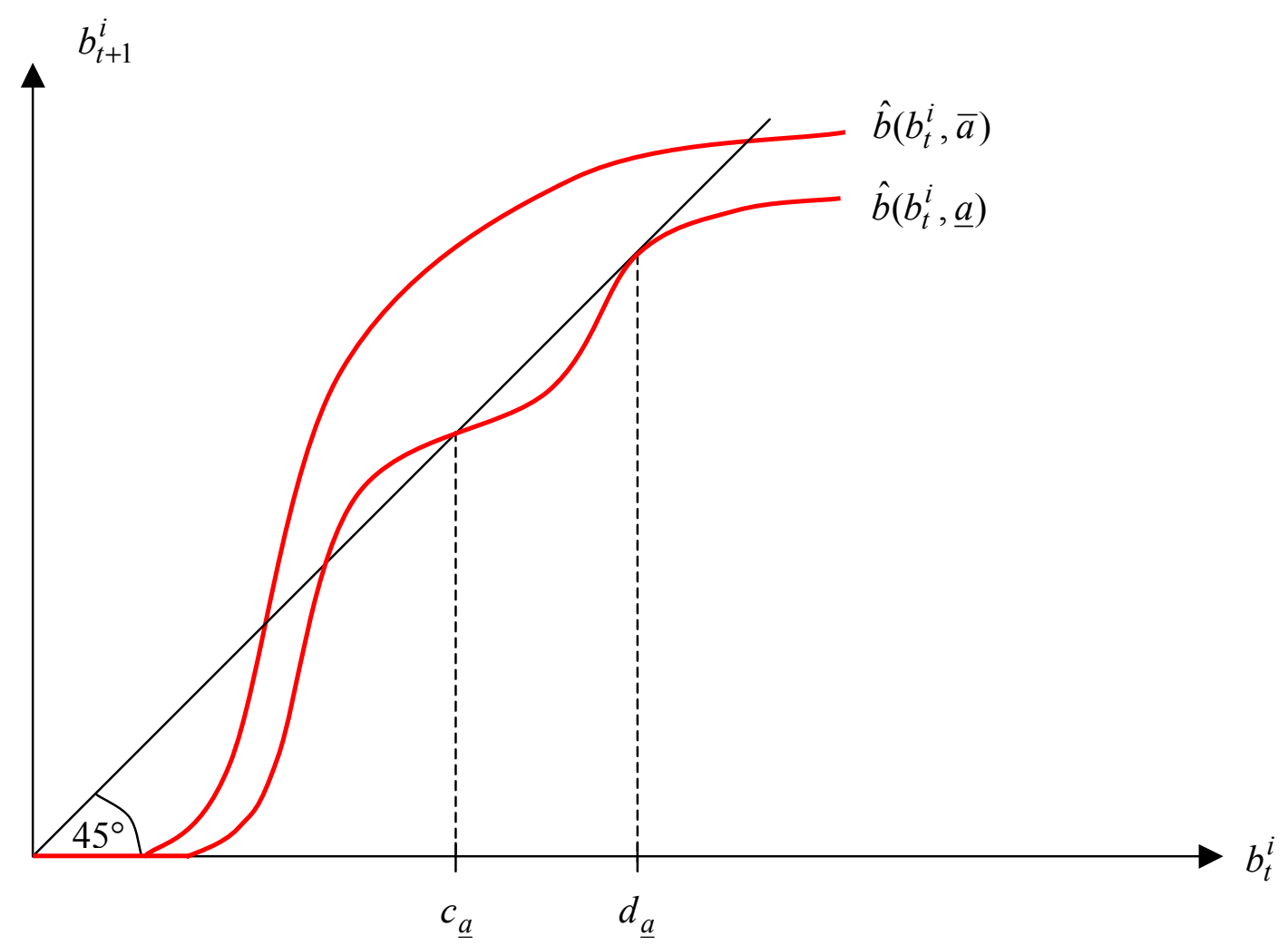

(c) 
Figure 3: Consistency with assumptions A6-A8.

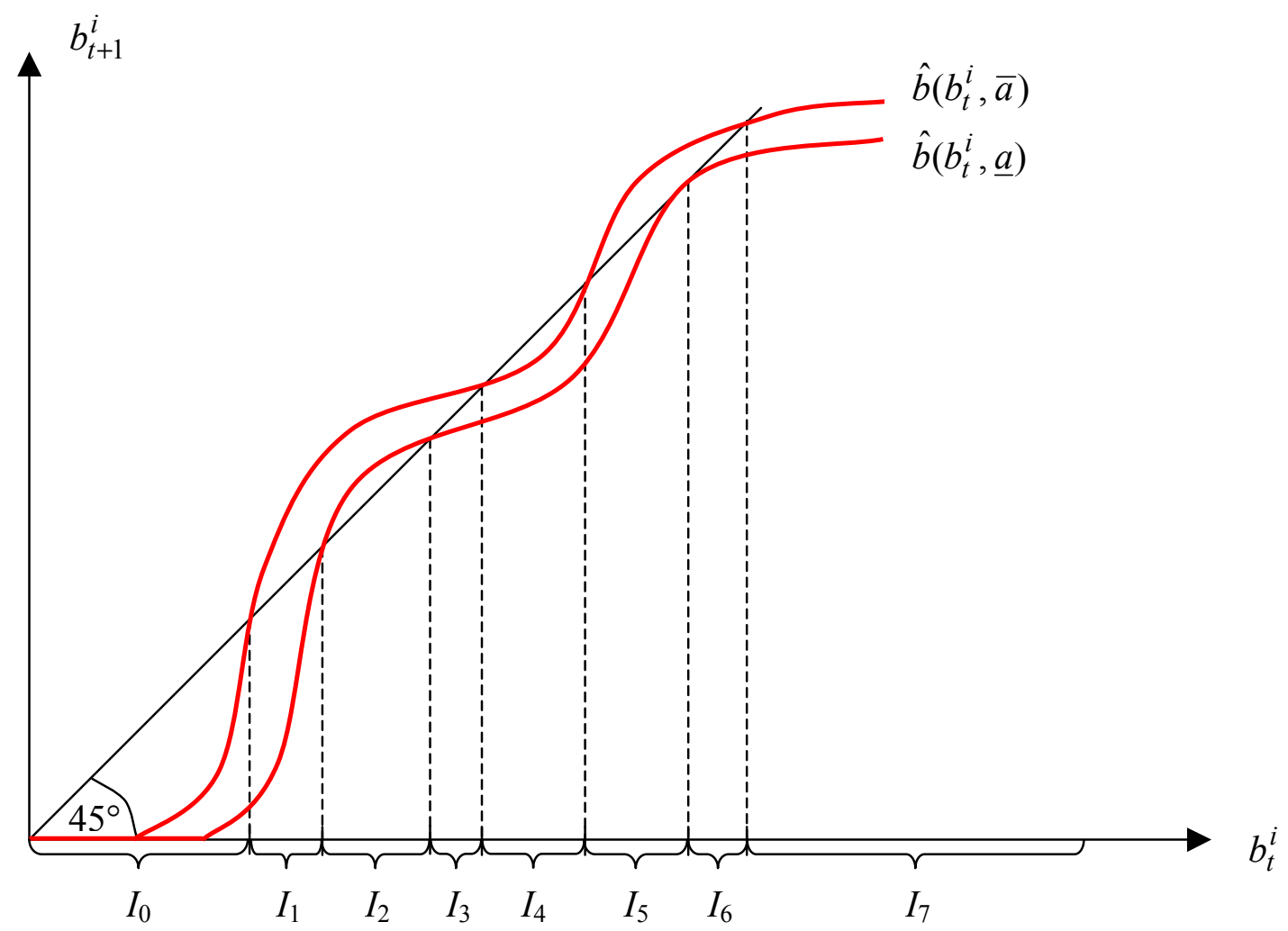

(a)

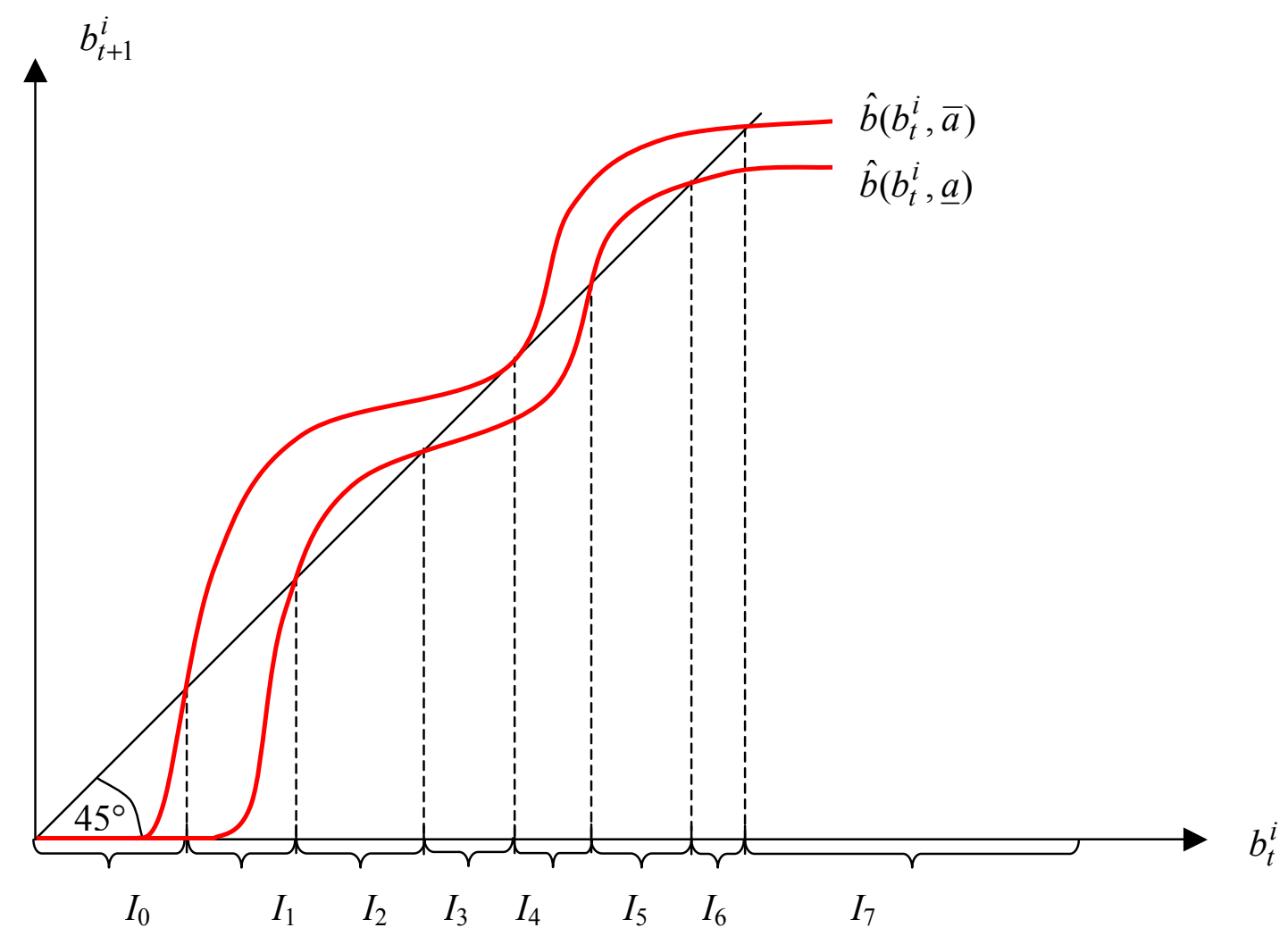

(b) 
(Figure 3 continued)

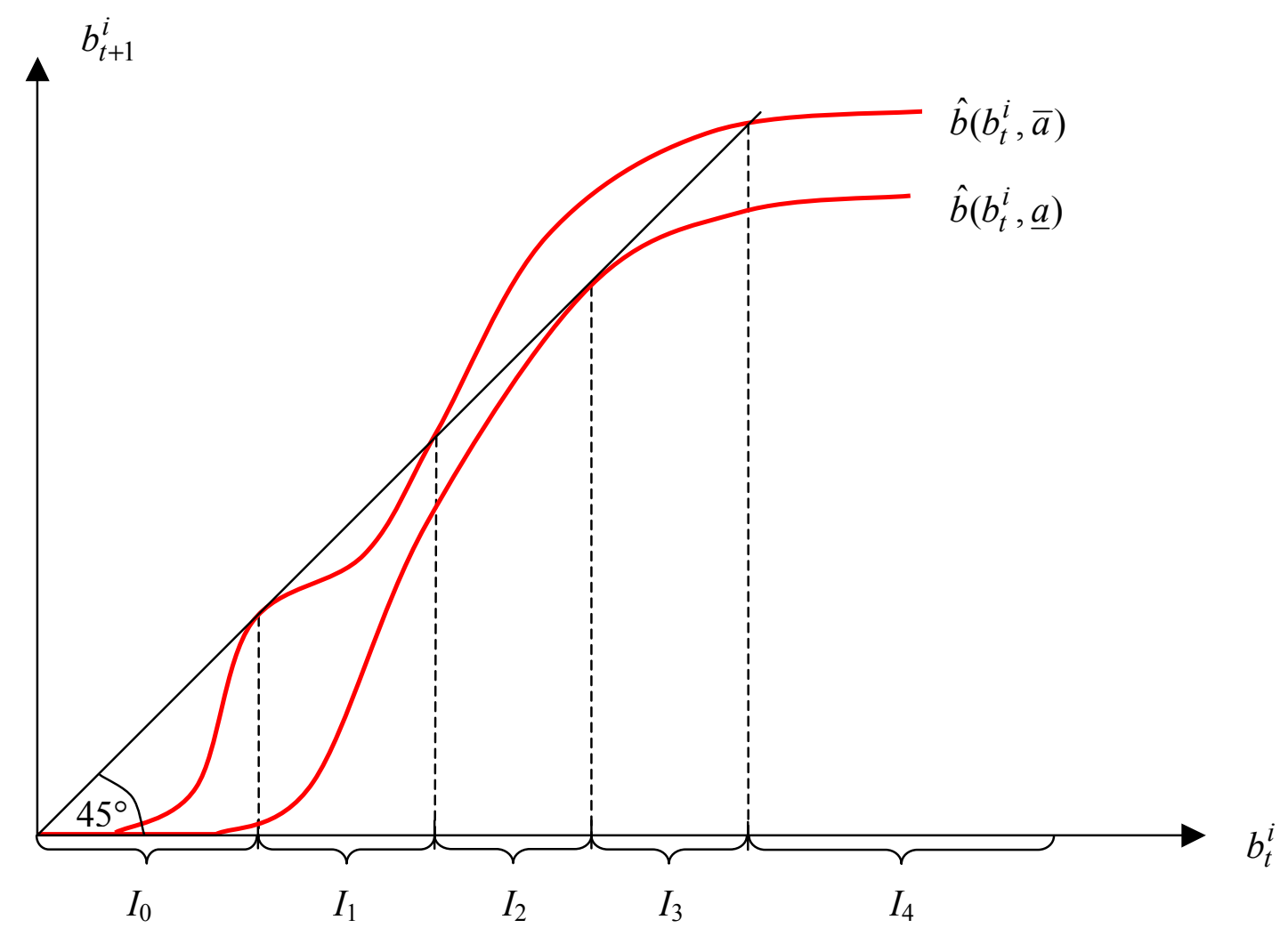

(c) 


\section{IZA Discussion Papers}

\begin{tabular}{|c|c|c|c|c|}
\hline No. & Author(s) & Title & Area & Date \\
\hline 941 & $\begin{array}{l}\text { R. V. Burkhauser } \\
\text { J. S. Butler } \\
\text { G. Gumus }\end{array}$ & $\begin{array}{l}\text { Option Value and Dynamic Programming Model } \\
\text { Estimates of Social Security Disability Insurance } \\
\text { Application Timing }\end{array}$ & 6 & $11 / 03$ \\
\hline 942 & $\begin{array}{l}\text { R. V. Burkhauser } \\
\text { J. S. Butler } \\
\text { G. Gumus }\end{array}$ & $\begin{array}{l}\text { Dynamic Modeling of the SSDI Application } \\
\text { Timing Decision: The Importance of Policy } \\
\text { Variables }\end{array}$ & 6 & $11 / 03$ \\
\hline 943 & $\begin{array}{l}\text { J. T. Addison } \\
\text { P. Teixeira }\end{array}$ & $\begin{array}{l}\text { What Have We Learned About the Employment } \\
\text { Effects of Severance Pay? Further Iterations of } \\
\text { Lazear et al. }\end{array}$ & 3 & $11 / 03$ \\
\hline 944 & $\begin{array}{l}\text { H. Görg } \\
\text { D. Greenaway }\end{array}$ & $\begin{array}{l}\text { Much Ado About Nothing? Do Domestic Firms } \\
\text { Really Benefit from Foreign Direct Investment? }\end{array}$ & 2 & $11 / 03$ \\
\hline 945 & $\begin{array}{l}\text { R. Schöb } \\
\text { D. E. Wildasin }\end{array}$ & $\begin{array}{l}\text { Economic Integration and Labor Market } \\
\text { Institutions: Worker Mobility, Earnings Risk, and } \\
\text { Contract Structure }\end{array}$ & 2 & $12 / 03$ \\
\hline 946 & M. Leonardi & $\begin{array}{l}\text { Earnings Instability of Job Stayers and Job } \\
\text { Changers }\end{array}$ & 1 & $12 / 03$ \\
\hline 947 & U. Sunde & $\begin{array}{l}\text { Potential, Prizes and Performance: Testing } \\
\text { Tournament Theory with Professional Tennis } \\
\text { Data }\end{array}$ & 7 & $12 / 03$ \\
\hline 948 & $\begin{array}{l}\text { A. Kugler } \\
\text { G. Pica }\end{array}$ & $\begin{array}{l}\text { Effects of Employment Protection and Product } \\
\text { Market Regulations on the Italian Labor Market }\end{array}$ & 6 & $12 / 03$ \\
\hline 949 & C. J. Flinn & $\begin{array}{l}\text { Minimum Wage Effects on Labor Market } \\
\text { Outcomes under Search with Bargaining }\end{array}$ & 6 & $12 / 03$ \\
\hline 950 & $\begin{array}{l}\text { P. Garibaldi } \\
\text { E. Wasmer }\end{array}$ & $\begin{array}{l}\text { Equilibrium Employment in a Model of Imperfect } \\
\text { Labor Markets }\end{array}$ & 1 & $12 / 03$ \\
\hline 951 & $\begin{array}{l}\text { P. Garibaldi } \\
\text { E. Wasmer }\end{array}$ & $\begin{array}{l}\text { Raising Female Employment: Reflexions and } \\
\text { Policy Tools }\end{array}$ & 5 & $12 / 03$ \\
\hline 952 & $\begin{array}{l}\text { O. Raaum } \\
\text { K. G. Salvanes } \\
\text { E. Ø. Sørensen }\end{array}$ & The Neighbourhood Is Not What It Used to Be & 3 & $12 / 03$ \\
\hline 953 & $\begin{array}{l}\text { O. Raaum } \\
\text { K. G. Salvanes } \\
\text { E. Ø. Sørensen }\end{array}$ & $\begin{array}{l}\text { The Impact of a Primary School Reform on } \\
\text { Educational Stratification: A Norwegian Study of } \\
\text { Neighbour and School Mate Correlations }\end{array}$ & 5 & $12 / 03$ \\
\hline 954 & $\begin{array}{l}\text { P. Portugal } \\
\text { J. T. Addison }\end{array}$ & Six Ways to Leave Unemployment & 6 & $12 / 03$ \\
\hline 955 & V. Grossmann & $\begin{array}{l}\text { Risky Human Capital Investment, Income } \\
\text { Distribution, and Macroeconomic Dynamics }\end{array}$ & 5 & $12 / 03$ \\
\hline
\end{tabular}

An updated list of IZA Discussion Papers is available on the center's homepage www.iza.org. 